\title{
Peridynamic Formulation for Higher Order Functionally Graded Beams
}

\author{
Zhenghao Yang, Erkan Oterkus* and Selda Oterkus
}

PeriDynamics Research Centre, Department of Naval Architecture, Ocean and Marine Engineering, University of Strathclyde, 100 Montrose Street, Glasgow G4 OLZ United Kingdom

\begin{abstract}
With the development of advanced manufacturing technologies, the importance of functionally graded materials is increasing due to their advantages over widely used traditional composites. In this study, a new higher order functionally graded beam formulation is presented by using peridynamics. Both thin and thick beams can be analysed by using the new formulation. Moreover, there is no need to use a shear correction factor. Euler-Lagrange equations and Taylor's expansion are utilised to derive the governing equations. Several benchmark problems are studied to validate the newly developed higher order functionally graded beam formulation by considering different boundary conditions including clamped, simply supported and mixed boundary conditions. Peridynamic results are compared against finite element analysis results. According to the comparison, peridynamic and finite element analysis results agree very well with each other.
\end{abstract}

Keywords: Peridynamics; Functionally graded; Higher order beam theory; Non-local; Euler-Lagrange equation

*Corresponding author: erkan.oterkus@strath.ac.uk 


\section{Nomenclature}

\begin{tabular}{|c|c|}
\hline$A$ & Cross-section Area \\
\hline $\boldsymbol{b}$ & Body force density vector \\
\hline$q$ & Generalized displacement vector \\
\hline$I$ & Second moment of cross-section area \\
\hline$I^{*} \& I^{* *}$ & Higher order moment of cross-section area \\
\hline$E$ & Young's Modulus \\
\hline$L$ & Lagrangian \\
\hline$U$ & Total potential Energy of the system \\
\hline$W$ & Strain energy density \\
\hline$V_{(k)}$ & Volume that contains material point $k$ \\
\hline$u$ & Axial displacement \\
\hline$u^{*}$ & Independent displacement variable that contributes axial deformation \\
\hline$v$ & Transverse displacement of material point $k$ \\
\hline$v^{*}$ & Independent displacement variable that contributes flexure deformation \\
\hline$\varphi$ & Rotational displacement \\
\hline$\varphi^{*}$ & Independent displacement variable that contributes flexure deformation \\
\hline$\varphi_{y}$ & Independent displacement variable that contributes axial deformation \\
\hline$\delta$ & PD horizon size \\
\hline$\mu$ & Shear modulus \\
\hline$\vartheta$ & Poisson's ratio \\
\hline$\xi_{(j)(k)}$ & The difference value of coordinate between material point $j$ and $k$ \\
\hline$(\cdot)^{(\alpha)},(\cdot)_{(\alpha)}$ & Physical quantity at material point $\alpha$ \\
\hline
\end{tabular}




\section{Introduction}

With the development of advanced manufacturing technologies, the importance of functionally graded materials is increasing due to their advantages over widely used traditional composites. According to functionally graded material concept, the material properties are continuously varying based on a certain distribution. Therefore, functionally graded materials do not suffer from stress concentrations in the thickness direction due to the laminated structure used for fiber reinforced composites. Functionally graded materials are usually made of a mixture of ceramic and metal to resist ultra-high-temperature environments and eliminate interface problems. Moreover, they are used in various engineering fields including aerospace, defence, electronics and biomedical industries to remove stress concentrations, reduce residual stresses and increase bond strength (Simsek, 2010). Significant amount of research studies are available in the literature focusing on the analysis of functionally graded materials including beam and plate formulations.

Amongst these Li (2008) developed a formulation to analyse functionally graded beams by including rotary inertia and shear deformation. Ebrahimi and Zia (2015) studied the functionally graded porous Timoshenko beams. Calim (2016) performed dynamic analysis of functionally graded Timoshenko beams with variable cross-section. Ashgari et. al. (2011) presented a size-dependent functionally graded Timoshenko beam formulation based on modified couple stress theory. Simsek (2010) investigated the dynamic behaviour of functionally graded Timoshenko beams subjected to a moving harmonic load. In another study, Tajalli et. al. (2013) derived a size-dependent formulation for functionally graded Timoshenko beams using strain gradient theory. Ebrahimi and Barati (2016) investigated the free vibration behaviour of functionally graded nanobeams utilizing third-order shear deformation beam theory. Kadoli et. al. (2008) used higher order shear deformation theory to examine the static characteristic of functionally graded metal-ceramic beams. Simsek and Reddy (2013) analysed functionally graded microbeams using 
modified couple stress theory and different higher order beam theories. Li and Batra (2013) derived analytical expressions of the critical buckling load of functionally graded Timoshenko beams subjected to different boundary conditions. Sun et. al. (2016) investigated buckling and post-buckling deformations of a functionally graded Timoshenko beam resting on an elastic foundation subjected to temperature rise using the shooting method. Pydah and Batra (2017) developed a shear deformation theory for thick circular beams which was used to analytically solve static deformations of bi-directional functionally graded circular beams. In another study, Fariborz and Batra (2019) performed free vibration analysis of functionally graded circular beams using a shear deformation theory by incorporating through-the-thickness logarithmic variation of the circumferential displacement and has a parabolic through-the-thickness distribution of shear strain. More recently, Menasria et. al. (2020) presented dynamic analysis of functionally graded sandwich plates rested on elastic foundation using refined shear deformation theory. Zine et al. (2020) investigated the bending response of functionally graded plates utilizing cubic shear deformation theory. Rabhi et. al. (2020) examined the buckling and vibration responses of exponentially graded sandwich plates resting on elastic medium using trigonometric shear deformation theory. Matouk et. al. (2020) analyzed the free vibrational behaviour of functionally graded nano-beams resting on elastic foundation exposed to hygro-thermal environment utilizing Timoshenko beam theory. Chikr et. al. (2020) and Refrafi et. al. (2020) performed buckling analysis of functionally graded sandwich plates based on a refined trigonometric shear deformation theory. Rahmani et. al. (2020) investigated the effect of boundary conditions on the bending and free vibration behaviour of functionally graded sandwich plates resting on elastic foundation using higher order shear deformation theory. Kaddari et. al. (2020) studied static and free vibration behavior of functionally graded porous plates resting on elastic foundation utilizing quasi-3D hyperbolic shear deformation theory. Tounsi et. al. (2020) developed a four-variable trigonometric integral shear deformation model for the hygro-thermo-mechanical analysis of functionally graded ceramic-metal plates resting on an elastic foundation. Boussoula et. al. (2020) performed 
thermomechanical analysis of functionally graded sandwich plates by employing nth-order shear deformation theory.

In this study, a new higher order functionally graded beam formulation is presented by using an alternative formulation, peridynamics, for the first time in the literature. The new formulation is suitable for both thin and thick plates, and do not require a shear correction factor as in Timoshenko beam formulation. Peridynamics (Silling, 2000; Oterkus et. al., 2012; Madenci and Oterkus, 2014; Wang et. al., 2018; Liu et. al., 2020; Yu and Li, 2020; Tong et. al., 2020) has governing equations in the form of integro-differential equations without containing any spatial derivatives. Therefore, it is especially suitable for predicting crack initiation and propagation inside the structures. Moreover, it has a parameter named horizon, which allows representation of non-classical structural and material behaviour that is usually observed at small scale structures. There exist various studies in the literature which present peridynamic Euler (O'Grady and Foster, 2014; Diyaroglu et. al., 2019) and Timoshenko beam (Diyaroglu et. al., 2015; Yang et. al., 2019) formulations. However, peridynamic formulation for higher order functionally graded beam is currently not available in the literature. To derive the equations of motion, Euler-Lagrange equation and Taylor's expansion are utilised. Several benchmark problems are considered to validate the current formulation by comparing against finite element analysis results.

\section{Classical Higher Order Beam Formulation for Functionally Graded Materials}

According to higher order beam theory, the motion of a material point can be represented as a function of material points along the central axis in $x y$ plane by using Taylor's expansion as

$$
\begin{aligned}
& u(x, y, t)=u(x, 0, t)+\left.y \frac{\partial u}{\partial y}\right|_{y=0}+\left.\frac{1}{2} y^{2} \frac{\partial^{2} u}{\partial y^{2}}\right|_{y=0}+\left.\frac{1}{3 !} y^{3} \frac{\partial^{3} u}{\partial y^{3}}\right|_{y=0}+\cdots \\
& v(x, y, t)=v(x, 0, t)+\left.y \frac{\partial v}{\partial y}\right|_{y=0}+\left.\frac{1}{2} y^{2} \frac{\partial^{2} v}{\partial y^{2}}\right|_{y=0}+\left.\frac{1}{3 !} y^{3} \frac{\partial^{3} v}{\partial y^{3}}\right|_{y=0}+\cdots
\end{aligned}
$$


The expressions given in Eqs. $(1 \mathrm{a}, \mathrm{b})$ can also be written by ignoring higher order terms as

$u(x, y, t)=u(x, 0, t)+y \varphi(x, t)+y^{2} u^{*}(x, t)+z^{3} \varphi^{*}(x, t)$

$v(x, y, t)=v(x, 0, t)+y \varphi_{y}(x, t)+y^{2} v^{*}(x, t)$

where $\varphi, u^{*}, \varphi^{*}, \varphi_{y}$ and $v^{*}$ are independent generalized displacements which are defined as

$\varphi(x, t)=\left.\frac{\partial u(x, y, t)}{\partial y}\right|_{y=0}$

$u^{*}(x, t)=\left.\frac{1}{2} \frac{\partial^{2} u(x, y, t)}{\partial y^{2}}\right|_{y=0}$

$\varphi^{*}(x, t)=\left.\frac{1}{3 !} \frac{\partial^{3} u(x, y, t)}{\partial y^{3}}\right|_{y=0}$

$\varphi_{y}(x, t)=\left.\frac{\partial v(x, y, t)}{\partial y}\right|_{y=0}$

$v^{*}(x, t)=\left.\frac{1}{2} \frac{\partial^{2} v(x, y, t)}{\partial y^{2}}\right|_{y=0}$

Hereafter, $u(x, 0, t), \varphi(x, t), u^{*}(x, t), \varphi^{*}(x, t), v(x, 0, t), \varphi_{y}(x, t)$ and $v^{*}(x, t)$ will be denoted as $u$, $\varphi, u^{*}, \varphi^{*}, v, \varphi_{y}$ and $v^{*}$, respectively.

Next, the strain components can be obtained by using Eqs. $(2 a, b)$ as

$\varepsilon_{x x}=\frac{\partial u}{\partial x}+y \frac{\partial \varphi}{\partial x}+y^{2} \frac{\partial u^{*}}{\partial x}+y^{3} \frac{\partial \varphi^{*}}{\partial x}$

$\varepsilon_{y y}=\varphi_{y}+2 y v^{*}$

$\gamma_{x y}=\left(\varphi+\frac{\partial v}{\partial x}\right)+y\left(2 u^{*}+\frac{\partial \varphi_{y}}{\partial x}\right)+y^{2}\left(3 \varphi^{*}+\frac{\partial v^{*}}{\partial x}\right)$

Unlike the strain-displacement relationships in the classical beam theories where the transverse normal is zero and the shearing strain varies linearly along the thickness, these relationships in higher order beam formulation are more realistic. Moreover, there is also no need to use a shear correction factor. 
The stress-strain relationships can be given for isotropic materials as

$\sigma_{x x}=\frac{E}{1-\vartheta^{2}}\left(\varepsilon_{x x}+\vartheta \varepsilon_{y y}\right)$

$\sigma_{z z}=\frac{E}{1-\vartheta^{2}}\left(\varepsilon_{y y}+\vartheta \varepsilon_{x x}\right)$

$\tau_{x y}=\mu \gamma_{x y}$

where $E=E(y), \mu=\mu(y)$ and $\vartheta=\vartheta(y)$ are the elastic and shear moduli, and Poisson's ratio, respectively, and they are function of the thickness coordinate, $y$.

The elastic strain energy density can be written as

$\widetilde{W}=\frac{1}{2}\left(\sigma_{x x} \varepsilon_{x x}+\sigma_{y y} \varepsilon_{y y}+\tau_{x y} \gamma_{x y}\right)$

Inserting Eqs. (5) into Eq. (6) results in

$\widetilde{W}=\frac{1}{2}\left[\frac{E}{1-\vartheta^{2}}\left(\varepsilon_{x x} \varepsilon_{x x}+\varepsilon_{y y} \varepsilon_{y y}+2 \vartheta \varepsilon_{y y} \varepsilon_{x x}\right)+\mu \gamma_{x y} \gamma_{x y}\right]$

Eq. (7) can also be expressed as a function of displacements by using Eqs. (4) as

$\widetilde{W}=\frac{1}{2}\left\{\frac{E}{1-\vartheta^{2}}\left[\left(\frac{\partial u}{\partial x}\right)^{2}+\left(\varphi_{y}\right)^{2}\right]+\frac{2 E y}{1-v^{2}}\left(\frac{\partial u}{\partial x} \frac{\partial \varphi}{\partial x}+2 \varphi_{y} v^{*}\right)+\frac{E y^{2}}{1-\vartheta^{2}}\left[\left(\frac{\partial \varphi}{\partial x}\right)^{2}+2 \frac{\partial u}{\partial x} \frac{\partial u^{*}}{\partial x}+4\left(v^{*}\right)^{2}\right]+\frac{2 E y^{3}}{1-\vartheta^{2}}\left(\frac{\partial u}{\partial x} \frac{\partial \varphi^{*}}{\partial x}+\right.\right.$ $\left.\left.\frac{\partial \varphi_{x}}{\partial x} \frac{\partial u^{*}}{\partial x}\right)+\frac{E y^{4}}{1-\vartheta^{2}}\left[\left(\frac{\partial u^{*}}{\partial x}\right)^{2}+2 \frac{\partial \varphi}{\partial x} \frac{\partial \varphi^{*}}{\partial x}\right]+\frac{2 E y^{5}}{1-\vartheta^{2}} \frac{\partial u^{*}}{\partial x} \frac{\partial \varphi^{*}}{\partial x}+\frac{E y^{6}}{1-\vartheta^{2}}\left(\frac{\partial \varphi^{*}}{\partial x}\right)^{2}\right\}+\left[\frac{E \vartheta}{1-\vartheta^{2}} \frac{\partial u}{\partial x} \varphi_{y}+\frac{E \vartheta y}{1-\vartheta^{2}}\left(2 \frac{\partial u}{\partial x} v^{*}+\frac{\partial \varphi}{\partial x} \varphi_{y}\right)+\right.$ $\left.\frac{E \vartheta y^{2}}{1-\vartheta^{2}}\left(2 \frac{\partial \varphi}{\partial x} v^{*}+\frac{\partial u^{*}}{\partial x} \varphi_{y}\right)+\frac{E \vartheta y^{3}}{1-\vartheta^{2}}\left(2 \frac{\partial u^{*}}{\partial x} v^{*}+\frac{\partial \varphi^{*}}{\partial x} \varphi_{y}\right)+\frac{2 E \vartheta y^{4}}{1-\vartheta^{2}} \frac{\partial \varphi^{*}}{\partial x} v^{*}\right]+\frac{1}{2}\left\{\mu\left(\varphi+\frac{\partial v}{\partial x}\right)^{2}+2 \mu y(\varphi+\right.$ $\left.\frac{\partial v}{\partial x}\right)\left(2 u^{*}+\frac{\partial \varphi_{y}}{\partial x}\right)+\mu y^{2}\left[\left(2 u^{*}+\frac{\partial \varphi_{y}}{\partial x}\right)^{2}+2\left(\varphi+\frac{\partial v}{\partial x}\right)\left(3 \varphi^{*}+\frac{\partial v^{*}}{\partial x}\right)\right]+2 \mu y^{3}\left(2 u^{*}+\frac{\partial \varphi_{y}}{\partial x}\right)\left(3 \varphi^{*}+\frac{\partial v^{*}}{\partial x}\right)+$ $\left.\mu y^{4}\left(3 \varphi^{*}+\frac{\partial v^{*}}{\partial x}\right)^{2}\right\}$

Integrating Eq. (8) over the cross-section $A$ gives the average cross-sectional strain energy density as

$\mathrm{W}=\frac{1}{A} \int_{A} \widetilde{W} d A=\frac{1}{2 A}\left\{\int_{A} \frac{E}{1-\vartheta^{2}} d A\left[\left(\frac{\partial u}{\partial x}\right)^{2}+\left(\varphi_{y}\right)^{2}\right]+\int_{A} \frac{2 E y}{1-\vartheta^{2}} d A\left(\frac{\partial u}{\partial x} \frac{\partial \varphi}{\partial x}+2 \varphi_{y} v^{*}\right)+\int_{A} \frac{E y^{2}}{1-\vartheta^{2}} d A\left[\left(\frac{\partial \varphi}{\partial x}\right)^{2}+\right.\right.$ $\left.2 \frac{\partial u}{\partial x} \frac{\partial u^{*}}{\partial x}+4\left(v^{*}\right)^{2}\right]+\int_{A} \frac{2 E y^{3}}{1-\vartheta^{2}} d A\left(\frac{\partial u}{\partial x} \frac{\partial \varphi^{*}}{\partial x}+\frac{\partial \varphi}{\partial x} \frac{\partial u^{*}}{\partial x}\right)+\int_{A} \frac{E y^{4}}{1-\vartheta^{2}} d A\left[\left(\frac{\partial u^{*}}{\partial x}\right)^{2}+2 \frac{\partial \varphi}{\partial x} \frac{\partial \varphi^{*}}{\partial x}\right]+$ 


$$
\begin{aligned}
& \left.\int_{A} \frac{2 E y^{5}}{1-\vartheta^{2}} d A \frac{\partial u^{*}}{\partial x} \frac{\partial \varphi^{*}}{\partial x}+\int_{A} \frac{E y^{6}}{1-\vartheta^{2}} d A\left(\frac{\partial \varphi^{*}}{\partial x}\right)^{2}\right\}+\frac{1}{A}\left[\int_{A} \frac{E \vartheta}{1-\vartheta^{2}} d A \frac{\partial u}{\partial x} \varphi_{y}+\int_{A} \frac{E \vartheta y}{1-\vartheta^{2}} d A\left(2 \frac{\partial u}{\partial x} v^{*}+\frac{\partial \varphi}{\partial x} \varphi_{y}\right)+\right. \\
& \left.\int_{A} \frac{E \vartheta y^{2}}{1-\vartheta^{2}} d A\left(2 \frac{\partial \varphi}{\partial x} v^{*}+\frac{\partial u^{*}}{\partial x} \varphi_{y}\right)+\int_{A} \frac{E \vartheta y^{3}}{1-\vartheta^{2}} d A\left(2 \frac{\partial u^{*}}{\partial x} v^{*}+\frac{\partial \varphi^{*}}{\partial x} \varphi_{y}\right)+\int_{A} \frac{2 E \vartheta y^{4}}{1-\vartheta^{2}} d A \frac{\partial \varphi^{*}}{\partial x} v^{*}\right]+\frac{1}{2 A}\left\{\int_{A} \mu d A(\varphi+\right. \\
& \left.\frac{\partial v}{\partial x}\right)^{2}+2 \int_{A} \mu y d A\left(\varphi+\frac{\partial v}{\partial x}\right)\left(2 u^{*}+\frac{\partial \varphi_{y}}{\partial x}\right)+\int_{A} \mu y^{2} d A\left[\left(2 u^{*}+\frac{\partial \varphi_{y}}{\partial x}\right)^{2}+2\left(\varphi+\frac{\partial v}{\partial x}\right)\left(3 \varphi^{*}+\frac{\partial v^{*}}{\partial x}\right)\right]+ \\
& \left.2 \int_{A} \mu y^{3} d A\left(2 u^{*}+\frac{\partial \theta_{y}}{\partial x}\right)\left(3 \varphi^{*}+\frac{\partial v^{*}}{\partial x}\right)+\int_{A} \mu y^{4} d A\left(3 \varphi^{*}+\frac{\partial v^{*}}{\partial x}\right)^{2}\right\}
\end{aligned}
$$

\section{Peridynamic Higher Order Beam Formulation for Functionally Graded Materials}

The PD equations of motion can be derived by utilizing Euler-Lagrange's equation as (Madenci and Oterkus, 2014):

$$
\frac{d}{d t} \frac{\partial L}{\partial \dot{\boldsymbol{q}}_{(k)}}-\frac{\partial L}{\partial \boldsymbol{q}_{(k)}}=0
$$

where $L=T-U$ is the Lagrangian and $\boldsymbol{q}$ is the generalized displacement vector which is defined as

$$
\mathbf{q}=\left(\begin{array}{lllllll}
u & \varphi & u^{*} & \varphi^{*} & v & \varphi_{y} & v^{*}
\end{array}\right)^{T}
$$

The total kinetic energy of the body, $T$ can be casted by utilizing Eqs. (2a) and (2b) as

$$
\begin{aligned}
& T=\frac{1}{2} \int_{0}^{L} \int_{A} \rho\left\{\dot{u}^{2}(x, y, t)+\dot{v}^{2}(x, y, t)\right\} d A d x=\frac{1}{2} \int_{0}^{L} \rho\left\{A\left(\dot{u}^{2}+\dot{v}^{2}\right)+I\left(\dot{\varphi}^{2}+2 \dot{u} \dot{u}^{*}+\dot{\varphi}_{y}^{2}+2 \dot{v} \dot{v}^{*}\right)+\right. \\
& \left.I^{*}\left[\left(\dot{u}^{*}\right)^{2}+2 \dot{\varphi} \dot{\varphi}^{*}+\left(\dot{v}^{*}\right)^{2}\right]+I^{* *}\left(\dot{\varphi}^{*}\right)^{2}\right\} d x
\end{aligned}
$$

where $I, I^{*}$ and $I^{* *}$ can be introduced as the second and higher moment of area, respectively, which are defined as

$$
\begin{aligned}
& I=\int_{A} y^{2} d A \\
& I^{*}=\int_{A} y^{4} d A
\end{aligned}
$$


Eq. (12) can be expressed in discretized form for the material point $k$ as

$$
\begin{aligned}
& T=\frac{1}{2} \sum_{k} \rho_{(k)}\left\{\left(\dot{u}_{(k)}^{2}+\dot{v}_{(k)}^{2}\right)+\frac{I_{(k)}}{A_{(k)}}\left[\dot{\varphi}_{(k)}^{2}+2 \dot{u}_{(k)} \dot{u}_{(k)}^{*}+\left(\dot{\varphi}_{y}^{(k)}\right)^{2}+2 \dot{v}_{(k)} \dot{v}_{(k)}^{*}\right]+\frac{I_{(k)}^{*}}{A_{(k)}}\left[\left(\dot{u}_{(k)}^{*}\right)^{2}+2 \dot{\varphi}_{(k)} \dot{\varphi}_{(k)}^{*}+\right.\right. \\
& \left.\left.\left(\dot{v}_{(k)}^{*}\right)^{2}\right]+\frac{I_{(k)}^{*}}{A_{(k)}}\left(\dot{\varphi}_{(k)}^{*}\right)^{2}\right\} V_{(k)}
\end{aligned}
$$

where $A_{(k)}$ is the area that contains the material point $k$.

The total potential energy stored in the body, $U$ can be obtained by summing potential energies of all material points including strain energy and energy due to external loads as

$$
U=\sum_{k}\left(W_{(k)}\left(\boldsymbol{q}_{(k)}, \boldsymbol{q}_{\left(1^{k}\right)}, \boldsymbol{q}_{\left(2^{k}\right)}, \ldots\right)-\boldsymbol{b}_{(k)} \boldsymbol{q}_{(k)}\right) V_{(k)}
$$

where $\boldsymbol{b}$ is the body load density vector

$$
\mathbf{b}=\left(\begin{array}{lllllll}
b_{u} & b_{\varphi} & 0 & 0 & b_{v} & 0 & 0
\end{array}\right)^{T}
$$

with $b_{u}, b_{\varphi}$ and $b_{v}$ represent the axial load, moment and transverse load densities, respectively.

Inserting Eqs. (14) and (15) into Eq. (10) yields:

$\rho_{(k)}\left\{\begin{array}{c}\ddot{u}_{(k)}+\frac{I_{(k)}}{A_{(k)}} \ddot{u}_{(k)}^{*} \\ \frac{I_{(k)}}{A_{(k)}} \ddot{\varphi}_{(k)}+\frac{I_{(k)}^{*}}{A_{(k)}} \ddot{\varphi}_{(k)}^{*} \\ \frac{I_{(k)}^{*}}{A_{(k)}} \ddot{u}_{(k)}^{* *}+\frac{I_{(k)}}{I_{(k)}} \ddot{u}_{(k)} \\ A_{(k)}^{*} \\ \ddot{\varphi}_{(k)}^{*}+\frac{I_{(k)}^{*}}{A_{(k)}} \ddot{\varphi}_{(k)}+\frac{I_{(k)}}{A_{(k)}} \ddot{v}^{*} \\ \frac{I_{(k)}}{A_{(k)}} \ddot{\varphi}_{y}^{(k)} \\ \frac{I_{(k)}^{*}}{A_{(k)}} \ddot{v}_{(k)}^{*}+\frac{I_{(k)}}{A_{(k)}} \ddot{v}_{(k)}\end{array}\right\} V_{(k)}=-\left\{\begin{array}{l}\frac{\partial W_{(k)}}{\partial u_{(k)}} V_{(k)}+\sum_{j} \frac{\partial W_{(j)}}{\partial u_{(k)}} V_{(j)} \\ \frac{\partial W_{(k)}}{\partial \varphi_{(k)}} V_{(k)}+\sum_{j} \frac{\partial W_{(j)}}{\partial \varphi_{(k)}} V_{(j)} \\ \frac{\partial W_{(k)}}{\partial u_{(k)}^{*}} V_{(k)}+\sum_{j} \frac{\partial W_{(j)}}{\partial u_{(k)}^{*}} V_{(j)} \\ \frac{\partial W_{(k)}}{\partial \varphi_{(k)}^{*}} V_{(k)}+\sum_{j} \frac{\partial W_{(j)}}{\partial \varphi_{(k)}^{*}} V_{(j)} \\ \frac{\partial W_{(k)}}{\partial v_{(k)}} V_{(k)}+\sum_{j} \frac{\partial W_{(j)}}{\partial v_{(k)}} V_{(j)} \\ \frac{\partial W_{(k)}}{\partial \varphi_{y}^{(k)}} V_{(k)}+\sum_{j} \frac{\partial W_{(j)}}{\partial \varphi_{y}^{(k)}} V_{(j)} \\ \frac{\partial W_{(k)}}{\partial v_{(k)}^{*}} V_{(k)}+\sum_{j} \frac{\partial W_{(j)}}{\partial v_{(k)}^{*}} V_{(j)}\end{array}\right\}+\left\{\begin{array}{c}b_{u}^{(k)} \\ b_{\varphi}^{(k)} \\ 0 \\ 0 \\ b_{v}^{(k)} \\ 0 \\ 0\end{array}\right\} V_{(k)}$ 
Strain energy density expression given in Eq. (9) can be written in PD Form by using the relationships given in Appendix A for the material point $k$ and its family member $j$ as

$$
\begin{aligned}
& W_{(k)}=\frac{1}{2} \frac{1}{\delta^{2} A^{2}}\left\{\int_{A} \frac{E}{1-\vartheta^{2}} d A \sum_{i}\left[\frac{\left(u_{\left(i^{k}\right)}-u_{(k)}\right)^{2}}{\left|\xi_{\left(i^{k}\right)(k)}\right|}+\left(\frac{\varphi_{y}^{\left(i^{k}\right)}+\varphi_{y}^{(k)}}{2}\right)^{2}\left|\xi_{\left(i^{k}\right)(k)}\right|\right] V_{\left(i^{k}\right)}+\right. \\
& \int_{A} \frac{2 E y}{1-\vartheta^{2}} d A \sum_{i}\left[\frac{\left(u_{\left(i^{k}\right)}-u_{(k)}\right)\left(\varphi_{\left(i^{k}\right)^{-}}\left(\varphi_{(k)}\right)\right.}{\left|\xi_{\left(i^{k}\right)(k)}\right|}+\left(\varphi_{y}^{\left(i^{k}\right)}+\varphi_{y}^{(k)}\right) \frac{v_{\left(i^{k}\right)}^{*}+v_{(k)}^{*}}{2}\left|\xi_{\left(i^{k}\right)(k)}\right|\right] V_{\left(i^{k}\right)}+ \\
& \int_{A} \frac{E y^{2}}{1-\vartheta^{2}} d A \sum_{i}\left[\frac{\left(\varphi_{\left(i^{k}\right)^{-}}[(k))^{2}\right.}{\left|\xi_{\left(i^{k}\right)(k)}\right|}+2 \frac{\left.\left(u_{\left(i^{k}\right)}-u_{(k)}\right)\left(u_{\left(i^{k}\right)}^{*}\right)_{(k)}^{*}\right)}{\left|\xi_{\left(i^{k}\right)(k)}\right|}+\left(v_{\left(i^{k}\right)}^{*}+v_{(k)}^{*}\right)^{2}\left|\xi_{\left(i^{k}\right)(k)}\right|\right] V_{\left(i^{k}\right)}+ \\
& \int_{A} \frac{2 E y^{3}}{1-\vartheta^{2}} d A \sum_{i}\left[\frac{\left(u_{\left(i^{k}\right)}-u_{(k)}\right)\left(\varphi_{\left(i^{k}\right)^{*}}^{*} \varphi_{(k)}^{*}\right)}{\left|\xi_{\left(i^{k}\right)(k)}\right|}+\frac{\left(\varphi_{\left(i^{k}\right)}-\varphi_{(k)}\right)\left(u_{\left(i^{k}\right)}^{*}-u_{(k)}^{*}\right)}{\left|\xi_{\left(i^{k}\right)(k)}\right|}\right] V_{\left(i^{k}\right)}+\int_{A} \frac{E y^{4}}{1-\vartheta^{2}} d A \sum_{i}\left[\frac{\left(u_{\left(i^{k}\right)}^{*}-u_{(k)}^{*}\right)^{2}}{\left|\xi_{\left(i^{k}\right)(k)}\right|}+\right. \\
& \left.2 \frac{\left(\varphi_{\left(i^{k}\right)}-\varphi_{(k)}\right)\left(\varphi_{\left(i^{k}\right)}^{*}-\varphi_{(k)}^{*}\right)}{\left|\xi_{\left(i^{k}\right)(k)}\right|}\right] V_{\left(i^{k}\right)}+\int_{A} \frac{2 E y^{5}}{1-\vartheta^{2}} d A \sum_{i} \frac{\left(u_{\left(i^{k}\right)}^{*}-u_{(k)}^{*}\right)\left(\varphi_{\left(i^{k}\right)}^{*}-\varphi_{(k)}^{*}\right)}{\left|\xi_{\left(i^{k}\right)(k)}\right|} V_{\left(i^{k}\right)}+ \\
& \left.\int_{A} \frac{E y^{6}}{1-\vartheta^{2}} d A \sum_{i} \frac{\left(\varphi_{\left(i^{k}\right)}^{*}-\varphi_{(k)}^{*}\right)^{2}}{\left|\xi_{\left(i^{k}\right)(k)}\right|} V_{\left(i^{k}\right)}\right\}+\frac{1}{\delta^{2} A^{2}}\left\{\int_{A} \frac{E \vartheta}{1-\vartheta^{2}} d A \sum_{i}\left(u_{\left(i^{k}\right)}-u_{(k)}\right) \frac{\varphi_{y}^{\left(i^{k}\right)}+\varphi_{y}^{(k)}}{2} \operatorname{sign}\left(\xi_{\left(i^{k}\right)(k)}\right) V_{\left(i^{k}\right)}+\right. \\
& \int_{A} \frac{E \vartheta y}{1-\vartheta^{2}} d A \sum_{i}\left[\left(u_{\left(i^{k}\right)}-u_{(k)}\right)\left(v_{\left(i^{k}\right)}^{*}+v_{(k)}^{*}\right) \operatorname{sign}\left(\xi_{\left(i^{k}\right)(k)}\right)+\left(\varphi_{\left(i^{k}\right)}-\right.\right. \\
& \left.\left.\varphi_{(k)}\right) \frac{\varphi_{y}^{\left(i^{k}\right)}+\varphi_{y}^{(k)}}{2} \operatorname{sign}\left(\xi_{\left(i^{k}\right)(k)}\right)\right] V_{\left(i^{k}\right)}+\int_{A} \frac{E \vartheta y^{2}}{1-\vartheta^{2}} d A \sum_{i}\left[\left(\varphi_{\left(i^{k}\right)}-\varphi_{(k)}\right)\left(v_{\left(i^{k}\right)}^{*}+v_{(k)}^{*}\right) \operatorname{sign}\left(\xi_{\left(i^{k}\right)(k)}\right)+\right. \\
& \left.\left(u_{\left(i^{k}\right)}^{*}-u_{(k)}^{*}\right) \frac{\varphi_{y}^{\left(i^{k}\right)}+\varphi_{y}^{(k)}}{2} \operatorname{sign}\left(\xi_{\left(i^{k}\right)(k)}\right)\right] V_{\left(i^{k}\right)}+\int_{A} \frac{E \vartheta y^{3}}{1-\vartheta^{2}} d A \sum_{i}\left[( u _ { ( i ^ { k } ) } ^ { * } - u _ { ( k ) } ^ { * } ) \left(v_{\left(i^{k}\right)}^{*}+\right.\right. \\
& \left.\left.v_{(k)}^{*}\right) \operatorname{sign}\left(\xi_{\left(i^{k}\right)(k)}\right)+\left(\varphi_{\left(i^{k}\right)}^{*}-\varphi_{(k)}^{*}\right) \frac{\varphi_{y}^{\left(i^{k}\right)}+\varphi_{y}^{(k)}}{2} \operatorname{sign}\left(\xi_{\left(i^{k}\right)(k)}\right)\right] V_{\left(i^{k}\right)}+\int_{A} \frac{2 E \vartheta y^{4}}{1-\vartheta^{2}} d A \sum_{i}\left(\varphi_{\left(i^{k}\right)}^{*}-\right.
\end{aligned}
$$

10 


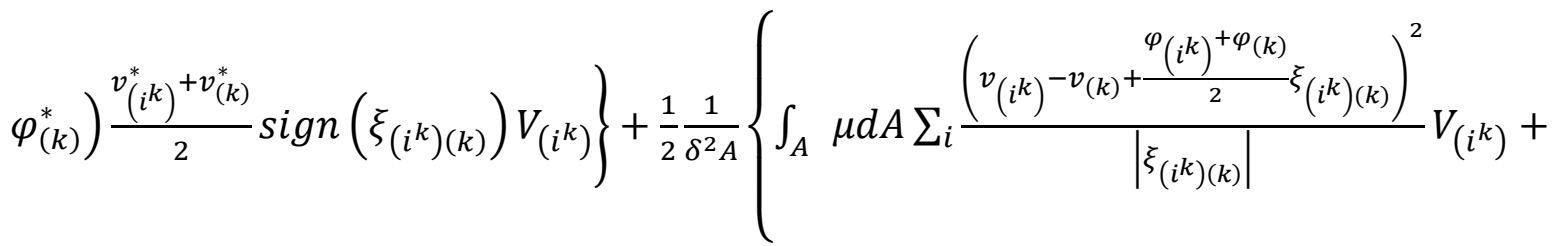

$2 \int_{A} \operatorname{GydA} \sum_{i}\left(v_{\left(i^{k}\right)}-v_{(k)}+\frac{\varphi_{\left(i^{k}\right)}+\varphi_{(k)}}{2} \xi_{\left(i^{k}\right)(k)}\right)\left(\frac{\varphi_{y}^{\left(i^{k}\right)}-\varphi_{y}^{(k)}}{\left|\xi_{\left(i^{k}\right)(k)}\right|}+\left(u_{\left(i^{k}\right)}^{*}+u_{(k)}^{*}\right) \operatorname{sign}\left(\xi_{\left(i^{k}\right)(k)}\right)\right) V_{\left(i^{k}\right)}+$

$\int_{A} \mu y^{2} d A \sum_{i} \frac{\left[\varphi_{y}^{\left(i^{k}\right)}-\varphi_{y}^{(k)}+\left(u_{\left(i^{k}\right)}^{*}+u_{(k)}^{*}\right) \xi_{\left(i^{k}\right)(k)}\right]^{2}+2\left(v_{\left(i^{k}\right)}-v_{(k)}+\frac{\varphi_{\left(i^{k}\right)}+\varphi_{(k)}}{2} \xi_{\left(i^{k}\right)(k)}\right)\left(v_{\left(i^{k}\right)}^{*}-v_{(k)}^{*}+3 \frac{\left.\varphi_{\left(i^{k}\right)}^{*}\right)^{+\varphi_{(k)}^{*}}}{2} \xi\left(i^{k}\right)(k)\right)}{\left|\xi_{\left(i^{k}\right)(k)}\right|} V_{\left(i^{k}\right)}+$

$2 \int_{A} \mu y^{3} d A \sum_{i} \frac{\varphi_{y}^{\left(i^{k}\right)}-\varphi_{y}^{(k)}+\left(u_{\left(i^{k}\right)}^{*}+u_{(k)}^{*}\right) \xi_{\left(i^{k}\right)(k)}}{\left|\xi_{\left(i^{k}\right)(k)}\right|}\left(v_{\left(i^{k}\right)}^{*}-v_{(k)}^{*}+3 \frac{\varphi_{\left(i^{k}\right)}^{*}+\varphi_{(k)}^{*}}{2} \xi_{\left(i^{k}\right)(k)}\right) V_{\left(i^{k}\right)}+$

$\left.\int_{A} \mu y^{4} d A \sum_{i} \frac{\left(v_{\left(i^{k}\right)}^{*}-v_{(k)}^{*}+3 \frac{\varphi_{\left(i^{k}\right)}^{*}+\varphi_{(k)}^{*}}{2} \xi_{\left(i^{k}\right)(k)}\right)^{2}}{\left|\xi_{\left(i^{k}\right)(k)}\right|} V_{\left(i^{k}\right)}\right\}$

$W_{(j)}=\frac{1}{2} \frac{1}{\delta^{2} A^{2}}\left\{\int_{A} \frac{E}{1-v^{2}} d A \sum_{i}\left[\frac{\left(u_{\left(i^{j}\right)}-u_{(j)}\right)^{2}}{\left|\xi_{\left(i^{j}\right)(j)}\right|}+\left(\frac{\varphi_{y}^{\left(i^{j}\right)}+\varphi_{y}^{(j)}}{2}\right)^{2}\left|\xi_{\left(i^{j}\right)(j)}\right|\right] V_{\left(i^{j}\right)}+\right.$

$\int_{A} \frac{2 E y}{1-\vartheta^{2}} d A \sum_{i}\left[\frac{\left(u_{\left(i^{j}\right)}-u_{(j)}\right)\left(\varphi_{\left(i^{j}\right)}-\varphi_{(j)}\right)}{\left|\xi_{(i j)(j)}\right|}+\left(\varphi_{y}^{\left(i^{j}\right)}+\varphi_{y}^{(j)}\right) \frac{v_{\left(i^{j}\right)}^{*}+v_{(j)}^{*}}{2}\left|\xi_{\left(i^{j}\right)(j)}\right|\right] V_{\left(i^{j}\right)}+$

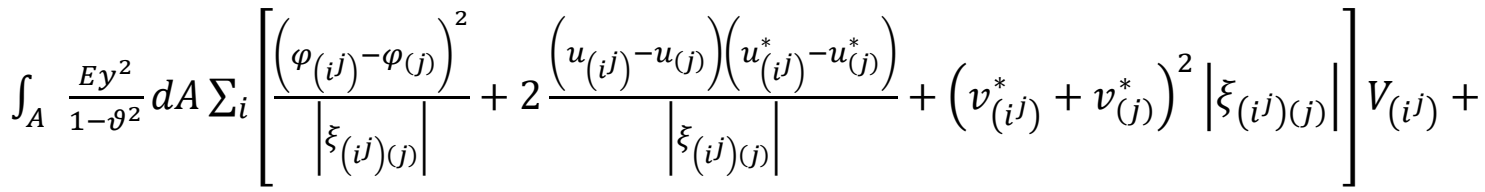

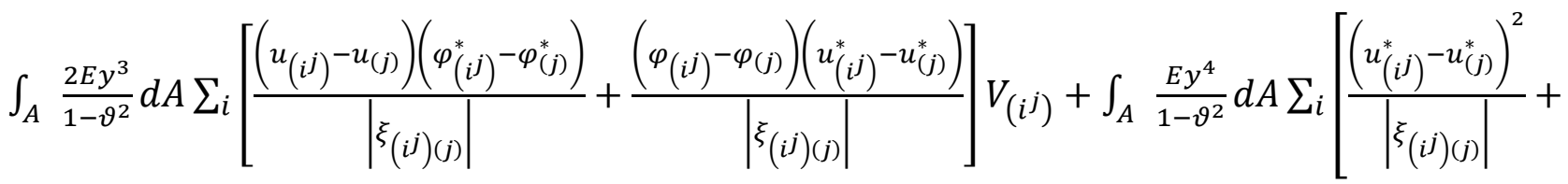

$\left.2 \frac{\left(\varphi_{(i j)}-\varphi_{(j)}\right)\left(\varphi_{(i j)}^{*}-\varphi_{(j)}^{*}\right)}{|\xi(i)(j)|}\right] V_{(i j)}+\int_{A} \frac{2 E y^{5}}{1-\vartheta^{2}} d A \sum_{i} \frac{\left(u_{(i j)}^{*}-u_{(j)}^{*}\right)\left(\varphi_{(i, j)}^{*}-\varphi_{(j)}^{*}\right)}{|\xi(i j)(j)|} V_{(i j)}+$

$\left.\int_{A} \frac{E y^{6}}{1-\vartheta^{2}} d A \sum_{i} \frac{\left(\varphi_{\left(i^{*}\right)}^{*}-\varphi_{(j)}^{*}\right)^{2}}{\mid \xi\left(i^{j}\right)(j)} V_{\left(i^{i}\right)}\right\}+\frac{1}{\delta^{2} A^{2}}\left\{\int_{A} \frac{E \vartheta}{1-\vartheta^{2}} d A \sum_{i}\left(u_{\left(i^{j}\right)}-u_{(j)}\right) \frac{\varphi_{y}^{(i j)}+\varphi_{y}^{(j)}}{2} \operatorname{sign}\left(\xi_{(i j)(j)}\right) V_{\left(i^{j}\right)}+\right.$ 
$\int_{Z} \frac{E \vartheta y}{1-\vartheta^{2}} d A \sum_{i}\left[\left(u_{\left(i^{j}\right)}-u_{(j)}\right)\left(v_{\left(i^{j}\right)}^{*}+v_{(j)}^{*}\right) \operatorname{sign}\left(\xi_{\left(i^{j}\right)(j)}\right)+\left(\varphi_{\left(i^{j}\right)}-\varphi_{(j)}\right) \frac{\varphi_{y}^{\left(i^{j}\right)}+\varphi_{y}^{(j)}}{2} \operatorname{sign}\left(\xi_{\left(i^{j}\right)(j)}\right)\right] V_{\left(i^{j}\right)}+$ $\int_{A} \frac{E \vartheta y^{2}}{1-\vartheta^{2}} d A \sum_{i}\left[\left(\varphi_{\left(i^{j}\right)}-\varphi_{(j)}\right)\left(v_{\left(i^{j}\right)}^{*}+v_{(j)}^{*}\right) \operatorname{sign}\left(\xi_{\left(i^{j}\right)(j)}\right)+\left(u_{\left(i^{j}\right)}^{*}-u_{(j)}^{*}\right) \frac{\varphi_{y}^{\left(i^{j}\right)}+\varphi_{y}^{(j)}}{2} \operatorname{sign}\left(\xi_{\left(i^{j}\right)(j)}\right)\right] V_{\left(i^{j}\right)}+$ $\int_{A} \frac{E \vartheta y^{3}}{1-\vartheta^{2}} d A \sum_{i}\left[\left(u_{\left(i^{j}\right)}^{*}-u_{(j)}^{*}\right)\left(v_{\left(i^{j}\right)}^{*}+v_{(j)}^{*}\right) \operatorname{sign}\left(\xi_{\left(i^{j}\right)(j)}\right)+\left(\varphi_{\left(i^{j}\right)}^{*}-\varphi_{(j)}^{*}\right) \frac{\varphi_{y}^{\left(i^{j}\right)}+\varphi_{y}^{(j)}}{2} \operatorname{sign}\left(\xi_{\left(i^{j}\right)(j)}\right)\right] V_{\left(i^{j}\right)}+$ $\left.\int_{A} \frac{2 E \vartheta y^{4}}{1-\vartheta^{2}} d A \sum_{i}\left(\varphi_{\left(i^{j}\right)}^{*}-\varphi_{(j)}^{*}\right) \frac{v_{\left(i^{j}\right)}^{*}+v_{(j)}^{*}}{2} \operatorname{sign}\left(\xi_{\left(i^{j}\right)(j)}\right) V_{\left(i^{j}\right)}\right\}+$

$\frac{1}{2} \frac{1}{\delta^{2} A^{2}}\left\{\int_{A} \mu d A \sum_{i} \frac{\left(v_{(i j)}-v_{(j)}+\frac{\left.\varphi_{(i j)}\right)^{+\varphi}(j)}{2} \xi_{(i j)(j)}\right)^{2}}{\left|\xi_{(i j)(k)}\right|} V_{\left(i^{j}\right)}+2 \int_{A} \mu y d A \sum_{i}\left(v_{(i j)}-v_{(j)}+\right.\right.$ $\left.\frac{\varphi_{(i j)} \varphi_{(j)}}{2} \xi_{\left(i^{j}\right)(j)}\right)\left(\frac{\varphi_{y}^{\left(i^{j}\right)}-\varphi_{y}^{(j)}}{\left|\xi_{\left(i^{j}\right)(j)}\right|}+\left(u_{\left(i^{j}\right)}^{*}+u_{(j)}^{*}\right) \operatorname{sign}\left(\xi_{\left(i^{j}\right)(j)}\right)\right) V_{\left(i^{j}\right)}+$

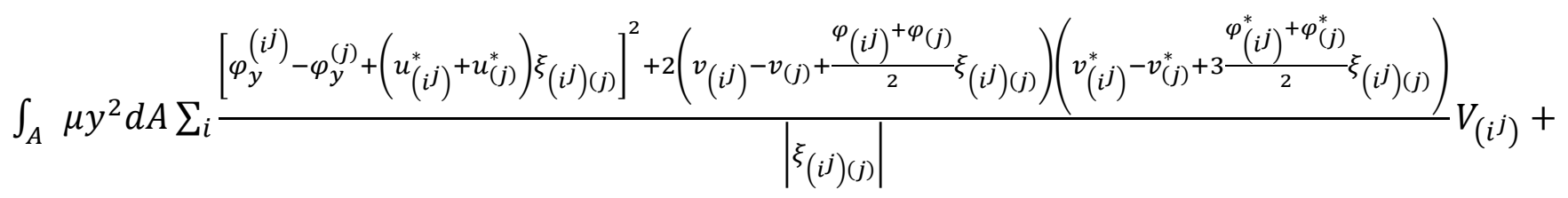
$2 \int_{A} \mu y^{3} d A \sum_{i} \frac{\left.\varphi_{y}^{\left(i^{j}\right)}-\varphi_{y}^{(j)}+\left(u_{(i j)}^{*}+u_{(j)}^{*}\right) \xi_{(i j)}\right)(j)}{\left|\xi_{(i j)}\right|(j) \mid}\left(v_{\left(i^{j}\right)}^{*}-v_{(j)}^{*}+3 \frac{\varphi_{\left(i^{j}\right)}^{*}+\varphi_{(j)}^{*}}{2} \xi_{\left(i^{j}\right)(j)}\right) V_{\left(i^{j}\right)}+$ $\left.\int_{A} \mu y^{4} d A \sum_{i} \frac{\left(v_{\left(i^{j}\right)}^{*}-v_{(j)}^{*}+3 \frac{\left.\varphi_{(i j)}^{*}\right)^{+\varphi_{(j)}^{*}}}{2} \xi_{(i j)(j)}\right)^{2}}{\left|\xi_{(i j)(j)}\right|} V_{\left(i^{j}\right)}\right\}$

with $\xi_{(\alpha)(\beta)}=x_{(\alpha)}-x_{(\beta)}$. Substituting Eqs. (18a) and (18b) into Eq. (17) results in the governing equations for higher order functionally graded beams as 


$$
\begin{aligned}
\rho_{(k)}\left(\ddot{u}_{(k)}+\frac{I}{A} \ddot{u}_{(k)}^{*}\right) & \\
& =\frac{2}{\delta^{2} A^{2}}\left[\int_{A} \frac{E}{1-\vartheta^{2}} d A \sum_{j} \frac{\mathrm{u}_{(j)}-\mathrm{u}_{(k)}}{\left|\xi_{(j)(k)}\right|} V_{(j)}+\int_{A} \frac{E \vartheta}{1-\vartheta^{2}} d A \sum_{j} \frac{\varphi_{y}^{(j)}+\varphi_{y}^{(k)}}{2} \operatorname{sign}\left(\xi_{(j)(k)}\right) V_{(j)}\right. \\
& +\int_{A} \frac{E y}{1-\vartheta^{2}} d A \sum_{j} \frac{\varphi_{(j)}-\varphi_{(k)}}{\left|\xi_{(j)(k)}\right|} V_{(j)}+\int_{A} \frac{E \vartheta y}{1-\varphi^{2}} d A \sum_{j}\left(v_{(j)}^{*}+v_{(k)}^{*}\right) \operatorname{sign}\left(\xi_{(j)(k)}\right) V_{(j)} \\
& \left.+\int_{A} \frac{E \mathrm{y}^{2}}{1-\vartheta^{2}} d A \sum_{j} \frac{\mathrm{u}_{(j)}^{*}-\mathrm{u}_{(k)}^{*}}{\left|\xi_{(j)(k)}\right|} V_{(j)}+\int_{A} \frac{E y^{3}}{1-\vartheta^{2}} d A \sum_{j} \frac{\varphi_{(j)}^{*}-\varphi_{(k)}^{*}}{\left|\xi_{(j)(k)}\right|} V_{(j)}\right]+b_{u}^{(k)}
\end{aligned}
$$

$$
\begin{aligned}
\rho_{(k)}\left(\frac{I}{A} \ddot{\varphi}_{(k)}+\right. & \left.\frac{I^{*}}{A} \ddot{\varphi}_{(k)}^{*}\right) \\
& =\frac{2}{\delta^{2} A^{2}}\left\{\int_{A} \frac{E y}{1-\vartheta^{2}} d A \sum_{j} \frac{u_{(j)}-u_{(k)}}{\left|\xi_{(j)(k)}\right|} V_{(j)}\right. \\
& +\int_{A} \frac{E \vartheta z}{1-\vartheta^{2}} d A \sum_{j} \frac{\varphi_{y}^{(j)}+\varphi_{y}^{(k)}}{2} \operatorname{sign}\left(\xi_{(j)(k)}\right) V_{(j)}+\int_{A} \frac{E y^{2}}{1-\vartheta^{2}} d A \sum_{j} \frac{\varphi_{(j)}-\varphi_{(k)}}{\left|\xi_{(j)(k)}\right|} V_{(j)} \\
& +\int_{A} \frac{E \vartheta y^{2}}{1-\vartheta^{2}} d A \sum_{j}\left(v_{(j)}^{*}+v_{(k)}^{*}\right) \operatorname{sign}\left(\xi_{(j)(k)}\right) V_{(j)}+\int_{A} \frac{E y^{3}}{1-\vartheta^{2}} d A \sum_{j} \frac{u_{(j)}^{*}-u_{(k)}^{*}}{\left|\xi_{(j)(k)}\right|} V_{(j)} \\
& +\int_{A} \frac{E y^{4}}{1-\vartheta^{2}} d A \sum_{i} \frac{\left.\varphi_{(j)}^{*}-\varphi_{(k)}^{*} V_{(j)}\right\}}{\left|\xi_{(j)(k)}\right|}{ } \frac{1}{\delta^{2} A^{2}}\left\{\int_{A} \mu d A \sum_{j}\left(v_{(j)}-v_{(k)}+\frac{\varphi_{(j)}+\varphi_{(k)}}{2} \xi_{(j)(k)}\right) \operatorname{sign}\left(\xi_{(j)(k)}\right) V_{(j)}\right. \\
& +\int_{A} \mu y d A \sum_{j}\left[\left(\varphi_{y}^{(j)}-\varphi_{y}^{(k)}\right) \operatorname{sign}\left(\xi_{(j)(k)}\right)+\left(u_{(j)}^{*}+u_{(k)}^{*}\right)\left|\xi_{(j)(k)}\right|\right] V_{(j)} \\
& \left.+\int_{A} \mu y^{2} d A \sum_{j}\left[\left(v_{(j)}^{*}-v_{(k)}^{*}\right) \operatorname{sign}\left(\xi_{(j)(k)}\right)+\frac{3}{2}\left(\varphi_{(j)}^{*}+\varphi_{(k)}^{*}\right)\left|\xi_{(j)(k)}\right|\right] V_{(j)}\right\}+b_{\varphi}^{(k)}
\end{aligned}
$$




$$
\begin{aligned}
\rho_{(k)}\left(\frac{I^{*}}{A} \ddot{u}_{(k)}^{*}+\right. & \left.\frac{I}{A} \ddot{u}_{(k)}\right) \\
& =\frac{2}{\delta^{2} A^{2}}\left\{\int_{A} \frac{E y^{2}}{1-\vartheta^{2}} d A \sum_{j} \frac{u_{(j)}-u_{(k)}}{\left|\xi_{(j)(k)}\right|} V_{(j)}\right. \\
& +\int_{A} \frac{E y^{2} \vartheta}{1-\vartheta^{2}} d A \sum_{j} \frac{\varphi_{y}^{(j)}+\varphi_{y}^{(k)}}{2} \operatorname{sign}\left(\xi_{(j)(k)}\right) V_{(j)}+\int_{A} \frac{E y^{3}}{1-\vartheta^{2}} d A \sum_{j} \frac{\varphi_{(j)}-\varphi_{(k)}}{\left|\xi_{(j)(k)}\right|} V_{(j)} \\
& +\int_{A} \frac{E \vartheta y^{3}}{1-\vartheta^{2}} d A \sum_{j}\left(v_{(j)}^{*}+v_{(k)}^{*}\right) \operatorname{sign}\left(\xi_{(j)(k)}\right) V_{(j)}+\int_{A} \frac{E y^{4}}{1-\vartheta^{2}} d A \sum_{j} \frac{u_{(j)}^{*}-u_{(k)}^{*}}{\left|\xi_{(j)(k)}\right|} V_{(j)} \\
& +\int_{A} \frac{E y^{5}}{1-\vartheta^{2}} d A \sum_{i} \frac{\left.\varphi_{(j)}^{*}-\varphi_{(k)}^{*} V_{(j)}\right\}}{\left|\xi_{(j)(k)}\right|} \\
& -\frac{2}{\delta^{2} A^{2}}\left\{\int_{A} \mu y d A \sum_{j}\left(v_{(j)}-v_{(k)}+\frac{\varphi_{(j)}+\varphi_{(k)}}{2} \xi_{(j)(k)}\right) \operatorname{sign}\left(\xi_{(j)(k)}\right) V_{(j)}\right. \\
& +\int_{A} \mu y^{2} d A \sum_{j}\left[\varphi_{y}^{(j)}-\varphi_{y}^{(k)}+\left(u_{(j)}^{*}+u_{(k)}^{*}\right) \xi_{(j)(k)}\right] \operatorname{sign}\left(\xi_{(j)(k)}\right) V_{(j)} \\
& \left.+\int_{A} \mu y^{3} d A \sum_{j}\left[\left(v_{(j)}^{*}-v_{(k)}^{*}\right) \operatorname{sign}\left(\xi_{(j)(k)}\right)+\frac{3}{2}\left(\varphi_{(j)}^{*}+\varphi_{(k)}^{*}\right)\left|\xi_{(j)(k)}\right|\right] V_{(j)}\right\}
\end{aligned}
$$




$$
\begin{aligned}
\rho_{(k)}\left(\frac{I^{* *}}{A} \ddot{\varphi}_{(k)}^{*}+\right. & \left.\frac{I^{*}}{A} \ddot{\varphi}_{(k)}\right) \\
& =\frac{2}{\delta^{2} A^{2}}\left\{\int_{A} \frac{E y^{3}}{1-\vartheta^{2}} d A \sum_{j} \frac{u_{(j)}-u_{(k)}}{\left|\xi_{(j)(k)}\right|} V_{(j)}\right. \\
& +\int_{A} \frac{E \vartheta y^{3}}{1-\vartheta^{2}} d A \sum_{j} \frac{\varphi_{y}^{(j)}+\varphi_{y}^{(k)}}{2} \operatorname{sign}\left(\xi_{(j)(k)}\right) V_{(j)}+\int_{A} \frac{E y^{4}}{1-\vartheta^{2}} d A \sum_{j} \frac{\varphi_{(j)}-\varphi_{(k)}}{\left|\xi_{(j)(k)}\right|} V_{(j)} \\
& +\int_{A} \frac{E \vartheta y^{4}}{1-\vartheta^{2}} d A \sum_{j}\left(v_{(j)}^{*}+v_{(k)}^{*}\right) \operatorname{sign}\left(\xi_{(j)(k)}\right) V_{(j)}+\int_{A} \frac{E y^{5}}{1-\vartheta^{2}} d A \sum_{j} \frac{u_{(j)}^{*}-u_{(k)}^{*}}{\left|\xi_{(j)(k)}\right|} V_{(j)} \\
& \left.+\int_{A} \frac{E y^{6}}{1-\vartheta^{2}} d A \sum_{j} \frac{\varphi_{(j)}^{*}-\varphi_{(k)}^{*}}{\left|\xi_{(j)(k)}\right|} V_{(j)}\right\} \\
& -\frac{3}{\delta^{2} A^{2}}\left\{\int_{A} \mu y^{2} d A \sum_{j}\left(v_{(j)}-v_{(k)}+\frac{\theta_{(j)}+\theta_{(k)}}{2} \xi_{(j)(k)}\right) \operatorname{sign}\left(\xi_{(j)(k)}\right) V_{(j)}\right. \\
& +\int_{A} \mu y^{3} d A \sum_{j}\left(\varphi_{y}^{(j)}-\varphi_{y}^{(k)}+\left(u_{(j)}^{*}+u_{(k)}^{*}\right) \xi_{(j)(k)}\right) \operatorname{sign}\left(\xi_{(j)(k)}\right) V_{(j)} \\
& \left.+\int_{A} \mu y^{4} d A \sum_{j}\left[v_{(j)}^{*}-v_{(k)}^{*}+\frac{3}{2}\left(\varphi_{(j)}^{*}+\varphi_{(k)}^{*}\right) \xi_{(j)(k)}\right] \operatorname{sign}\left(\xi_{(j)(k)}\right) V_{(j)}\right\}
\end{aligned}
$$

$$
\begin{aligned}
& \rho_{(k)}\left(\ddot{v}_{(k)}+\frac{I}{A} \ddot{v}^{*}\right) \\
&=\frac{2}{\delta^{2} A^{2}}\left[\int_{A} \mu d A \sum_{j} \frac{v_{(j)}-v_{(k)}}{\left|\xi_{(j)(k)}\right|}+\frac{\varphi_{(j)}+\varphi_{(k)}}{2} \operatorname{sign}\left(\xi_{(j)(k)}\right) V_{(j)}\right. \\
&+\int_{A} \mu y d A \sum_{j}\left(\frac{\varphi_{y}^{(j)}-\varphi_{y}^{(k)}}{\left|\xi_{(j)(k)}\right|}+\left(u_{(j)}^{*}+u_{(k)}^{*}\right) \operatorname{sign}\left(\xi_{(j)(k)}\right)\right) V_{(j)} \\
&\left.+\int_{A} \mu y^{2} d A \sum_{j}\left(\frac{v_{(j)}^{*}-v_{(k)}^{*}}{\left|\xi_{(j)(k)}\right|}+\frac{3}{2}\left(\theta_{(j)}^{*}+\theta_{(k)}^{*}\right) \operatorname{sign}\left(\xi_{(j)(k)}\right)\right) V_{(j)}\right]+b_{v}^{(k)}
\end{aligned}
$$




$$
\begin{aligned}
& \rho_{(k)} \frac{I}{A} \ddot{\varphi}_{z}^{(k)}=-\frac{2}{\delta^{2} A^{2}}\left[\frac{1}{2} \int_{A} \frac{E}{1-\vartheta^{2}} d A \sum_{j} \frac{\varphi_{y}^{(j)}+\varphi_{y}^{(k)}}{2}\left|\xi_{(j)(k)}\right| V_{(j)}\right. \\
& +\frac{1}{2} \int_{A} \frac{E \vartheta}{1-\vartheta^{2}} d A \sum_{j}\left(u_{(j)}-u_{(k)}\right) \operatorname{sign}\left(\xi_{(j)(k)}\right) V_{(j)} \\
& +\int_{A} \frac{E y \vartheta}{1-\vartheta^{2}} d A \sum_{j} \frac{\varphi_{(j)}-\varphi_{(k)}}{2} \operatorname{sign}\left(\xi_{(j)(k)}\right) V_{(j)} \\
& +\int_{A} \frac{E y}{1-\vartheta^{2}} d A \sum_{j} \frac{v_{(j)}^{*}+v_{(k)}^{*}}{2}\left|\xi_{(j)(k)}\right| V_{(j)} \\
& +\int_{A} \frac{E \vartheta \mathrm{y}^{2}}{1-\vartheta^{2}} d A \sum_{j} \frac{u_{(j)}^{*}-u_{(k)}^{*}}{2} \operatorname{sign}\left(\xi_{(j)(k)}\right) V_{(j)} \\
& \left.+\int_{A} \frac{E \vartheta y^{3}}{1-\vartheta^{2}} d A \sum_{j} \frac{\varphi_{(j)}^{*}-\varphi_{(k)}^{*}}{2} \operatorname{sign}\left(\xi_{(j)(k)}\right) V_{(j)}\right] \\
& +\frac{2}{\delta^{2} A^{2}}\left[\int_{A} \mu y d A \sum_{j}\left(\frac{v_{(j)}-v_{(k)}}{\left|\xi_{(j)(k)}\right|}+\frac{\varphi_{(j)}+\varphi_{(k)}}{2} \operatorname{sign}\left(\xi_{(j)(k)}\right)\right) V_{\left(i^{k}\right)}\right. \\
& +\int_{A} \mu y^{2} d A \sum_{j} \frac{\varphi_{y}^{(j)}-\varphi_{y}^{(k)}+\left(\mathrm{u}_{(j)}^{*}+\mathrm{u}_{(k)}^{*}\right) \xi_{(j)(k)}}{\left|\xi_{(j)(k)}\right|} V_{(j)} \\
& \left.+\int_{A} \mu y^{3} d A \sum_{i}\left(\frac{v_{(j)}^{*}-v_{(k)}^{*}}{\left|\xi_{(j)(k)}\right|}+\frac{3}{2}\left(\varphi_{(j)}^{*}+\varphi_{(k)}^{*}\right) \operatorname{sign}\left(\xi_{(j)(k)}\right)\right) V_{(j)}\right]
\end{aligned}
$$




$$
\begin{aligned}
& \rho_{(k)}\left(\frac{I^{*}}{A} \ddot{v}_{(k)}^{*}+\frac{I}{A} \ddot{v}_{(k)}\right) \\
& =-\frac{2}{\delta^{2} A^{2}}\left\{\int_{A} \frac{E y}{1-\vartheta^{2}} d A \sum_{j} \frac{\varphi_{y}^{(j)}+\varphi_{y}^{(k)}}{2}\left|\xi_{(j)(k)}\right| V_{(j)}\right. \\
& +\int_{A} \frac{E y \vartheta}{1-\vartheta^{2}} d A \sum_{j}\left(u_{(j)}-u_{(k)}\right) \operatorname{sign}\left(\xi_{(j)(k)}\right) V_{(j)} \\
& +\int_{A} \frac{E \mathrm{y}^{2}}{1-\vartheta^{2}} d A \sum_{j}\left(v_{(j)}^{*}+v_{(k)}^{*}\right)\left|\xi_{(j)(k)}\right| V_{(j)} \\
& +\int_{A} \frac{E \vartheta \mathrm{y}^{2}}{1-\vartheta^{2}} d A \sum_{j}\left(\varphi_{(j)}-\varphi_{(k)}\right) \operatorname{sign}\left(\xi_{(j)(k)}\right) V_{(j)} \\
& +\int_{A} \frac{E \vartheta y^{3}}{1-\vartheta^{2}} d A \sum_{j}\left(u_{(j)}^{*}-u_{(k)}^{*}\right) \operatorname{sign}\left(\xi_{(j)(k)}\right) V_{(j)} \\
& \left.+\int_{A} \frac{E \vartheta y^{4}}{1-\vartheta^{2}} d A \sum_{j}\left(\varphi_{(j)}^{*}-\varphi_{(k)}^{*}\right) \operatorname{sign}\left(\xi_{(j)(k)}\right) V_{(j)}\right\} \\
& +\frac{2}{\delta^{2} A^{2}}\left\{\int_{A} \mu y^{2} d A \sum_{j}\left(\frac{v_{(j)}-v_{(k)}}{\left|\xi_{(j)(k)}\right|}+\frac{\varphi_{(j)}+\varphi_{(k)}}{2} \operatorname{sign}\left(\xi_{(j)(k)}\right)\right) V_{(j)}\right. \\
& +\int_{A} \mu y^{3} d A \sum_{j}\left(\frac{\varphi_{y}^{(j)}-\theta_{y}^{(k)}}{\left|\xi_{(j)(k)}\right|}+\left(u_{(j)}^{*}+u_{(k)}^{*}\right) \operatorname{sign}\left(\xi_{(j)(k)}\right)\right) V_{(j)} \\
& \left.+\int_{A} \mu y^{4} d A \sum_{j} \frac{v_{(j)}^{*}-v_{(k)}^{*}}{\left|\xi_{(j)(k)}\right|}+\frac{3}{2}\left(\varphi_{(j)}^{*}+\varphi_{(k)}^{*}\right) \operatorname{sign}\left(\xi_{(j)(k)}\right) V_{(j)}\right\}
\end{aligned}
$$

\section{Numerical Results}

For the numerical results, the variation of the functionally graded material properties is defined as

$$
\begin{aligned}
& E(y)=\left(E_{\text {Top }}-E_{\text {Bottom }}\right) \frac{y}{h}+\frac{1}{2}\left(E_{\text {Top }}+E_{\text {Bottom }}\right)(G P a) \\
& \mu(y)=\frac{E(y)}{2(1+0.3)} \\
& \mu(y)=0.3
\end{aligned}
$$


where $h$ represents the thickness of the beam. The length, thickness and width of the beam are specified as $L=1 \mathrm{~m}, h=0.15 \mathrm{~m}$ and $b=0.1 \mathrm{~m}$, respectively. The Young's modulus at the top surface is $E_{\text {Top }}=$ $200 \mathrm{GPa}$ and at the bottom surface is $E_{\text {Bottom }}=100 \mathrm{GPa}$. For the PD model, the discretization size is specified as $\Delta x=0.002 \mathrm{~m}$ with a horizon size of $\delta=3 \Delta x$.

\subsection{Simply Supported Beam Subjected to Transverse Loading}

In the first numerical example, a simply supported functionally graded beam is considered as shown in Fig. 1. The beam is subjected to a concentrated force of $F_{y}=1000 \mathrm{~N}$ at its center.

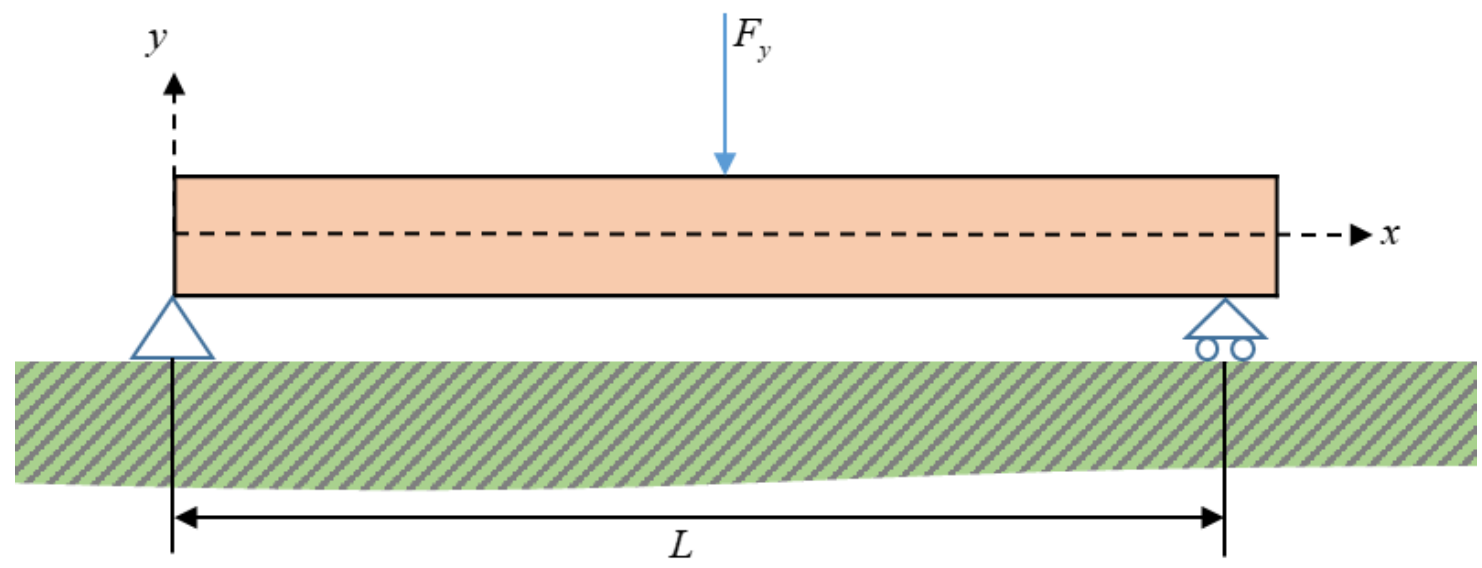

Figure 1. Simply supported beam subjected to transverse loading.

To impose simply supported boundary conditions, a fictitious region with a size of horizon is introduced outside of the actual domain with the following conditions:

$$
\begin{array}{ll}
v_{\left(i^{*}\right)}=-v_{(i)} & , \quad v_{\left(i^{*}\right)}^{*}=-v_{(i)}^{*} \\
u_{\left(i^{*}\right)}=-u_{(i)} & , \quad u_{\left(i^{*}\right)}^{*}=-u_{(i)}^{*} \\
\varphi_{\left(i^{*}\right)}=\varphi_{(i)} & , \quad \varphi_{\left(i^{*}\right)}^{*}=\varphi_{(i)}^{*} \\
\varphi_{y}^{\left(i^{*}\right)}=-\varphi_{y}^{(i)} & \text { for } i=1,2,3
\end{array}
$$


where $i$ and $i^{*}$ indices denote the three material points inside the actual and fictitious regions, respectively, around the constrained boundary.

On the other hand, roller support boundary condition is applied by introducing a fictitious region with a size of horizon with the following conditions:

$v_{\left(i^{*}\right)}=-v_{(i)} \quad, \quad v_{\left(i^{*}\right)}^{*}=-v_{(i)}^{*}$

$u_{\left(i^{*}\right)}=u_{(i)} \quad, \quad u_{\left(i^{*}\right)}^{*}=u_{(i)}^{*}$

$\varphi_{\left(i^{*}\right)}=\varphi_{(i)} \quad, \quad \varphi_{\left(i^{*}\right)}^{*}=\varphi_{(i)}^{*}$

$\varphi_{y}^{\left(i^{*}\right)}=\varphi_{y}^{(i)} \quad$ for $i=1,2,3$

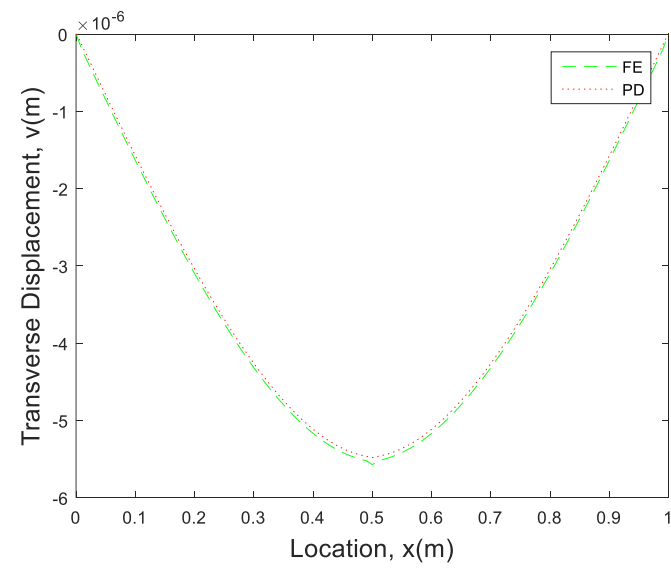

(a)

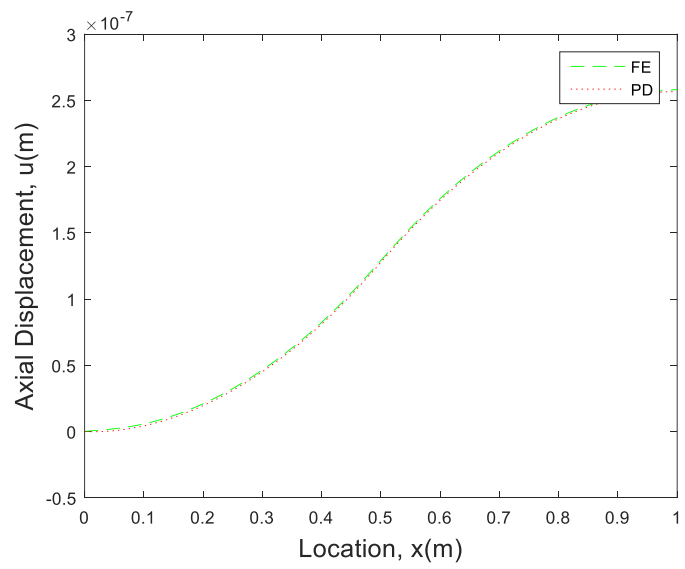

(b)

Figure 2. Variation of (a) transverse displacement, (b) axial displacement along the beam.

As shown in Fig. 2, PD solutions are compared against finite element (FE) analysis results obtained by using ANSYS. For both transverse and axial displacements along the beam, there is a very good agreement between PD and FE results. The maximum difference between PD and FE results is $1.7 \%$ which can be 
further reduced by increasing the numerical of material points in the PD solution domain. Moreover, simply-supported boundary conditions are successfully represented in the current model.

\subsection{Cantilever beam subjected to a point end load}

In the second example, a cantilever functionally graded beam is considered as shown in Fig. 3 . The beam is subjected to a concentrated force of $F_{y}=1000 \mathrm{~N}$ at the free end.

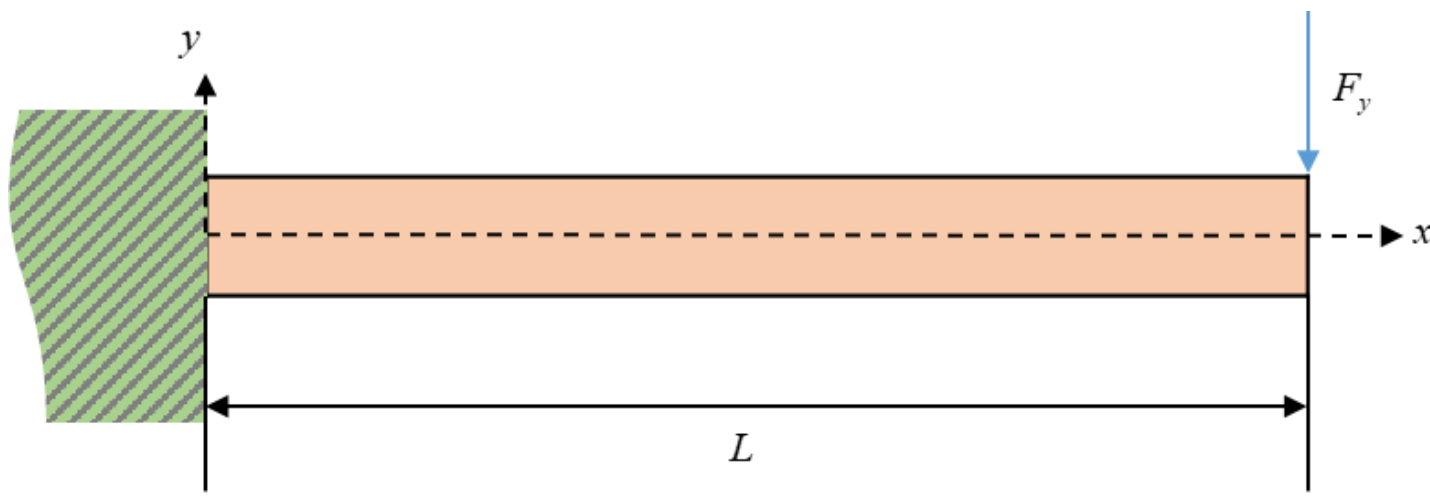

Figure 3. Cantilever beam subjected to a point end load.

To impose clamped boundary conditions, a fictitious region with a size of horizon is introduced with the following conditions:

$v_{\left(i^{*}\right)}=v_{(i)} \quad, \quad v_{\left(1^{*}\right)}=v_{(1)}=0$

$v_{\left(i^{*}\right)}^{*}=v_{(i)}^{*} \quad, \quad v_{\left(1^{*}\right)}^{*}=v_{(1)}^{*}=0$

$u_{\left(i^{*}\right)}=-u_{(i)} \quad, \quad u_{\left(i^{*}\right)}^{*}=-u_{(i)}^{*}$

$\varphi_{\left(i^{*}\right)}=-\varphi_{(i)} \quad, \quad \varphi_{\left(i^{*}\right)}^{*}=-\varphi_{(i)}^{*}$

$\varphi_{y}^{\left(i^{*}\right)}=-\varphi_{y}^{(i)} \quad$ for $i=1,2,3$ 


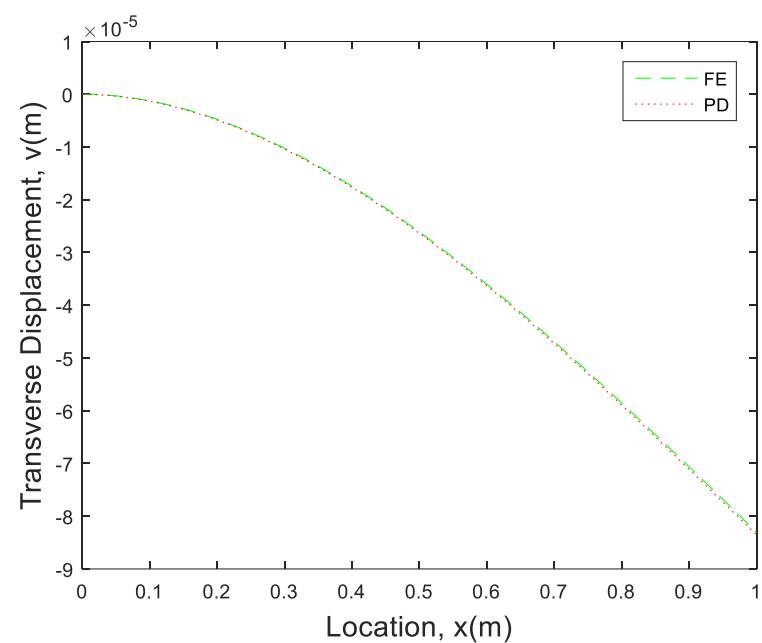

(a)

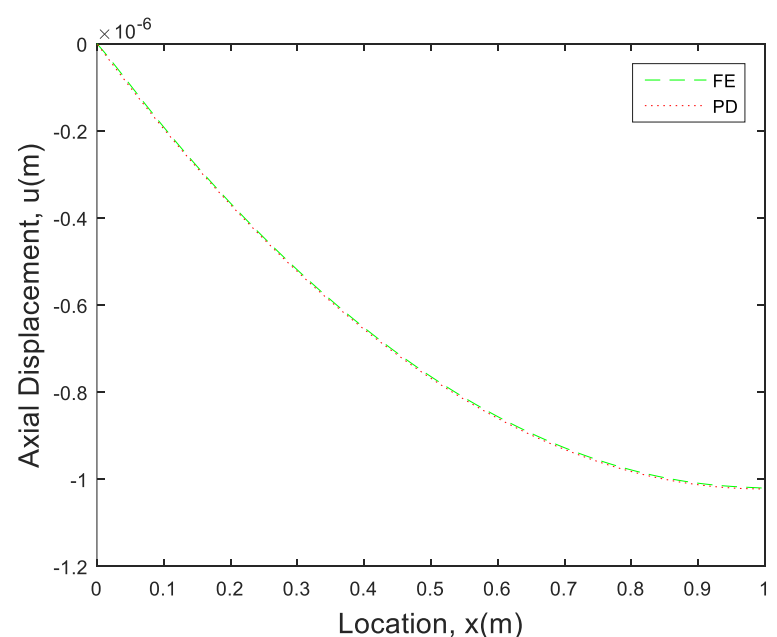

(b)

Figure 4. Variation of (a) transverse displacement, (b) axial displacement along the beam.

As shown in Fig. 4, a very good agreement is observed between PD and FE results for both transverse and axial displacement results. The maximum difference between PD and FE results is $0.5 \%$. Moreover, clamped boundary conditions are successfully represented in the current model.

\subsection{Clamped-roller supported beam subjected to transverse loading}

In the final example, a clamped-roller supported functionally graded beam is considered as shown in Fig.

5. The beam is subjected to a concentrated force of $F_{y}=1000 \mathrm{~N}$ at its center.

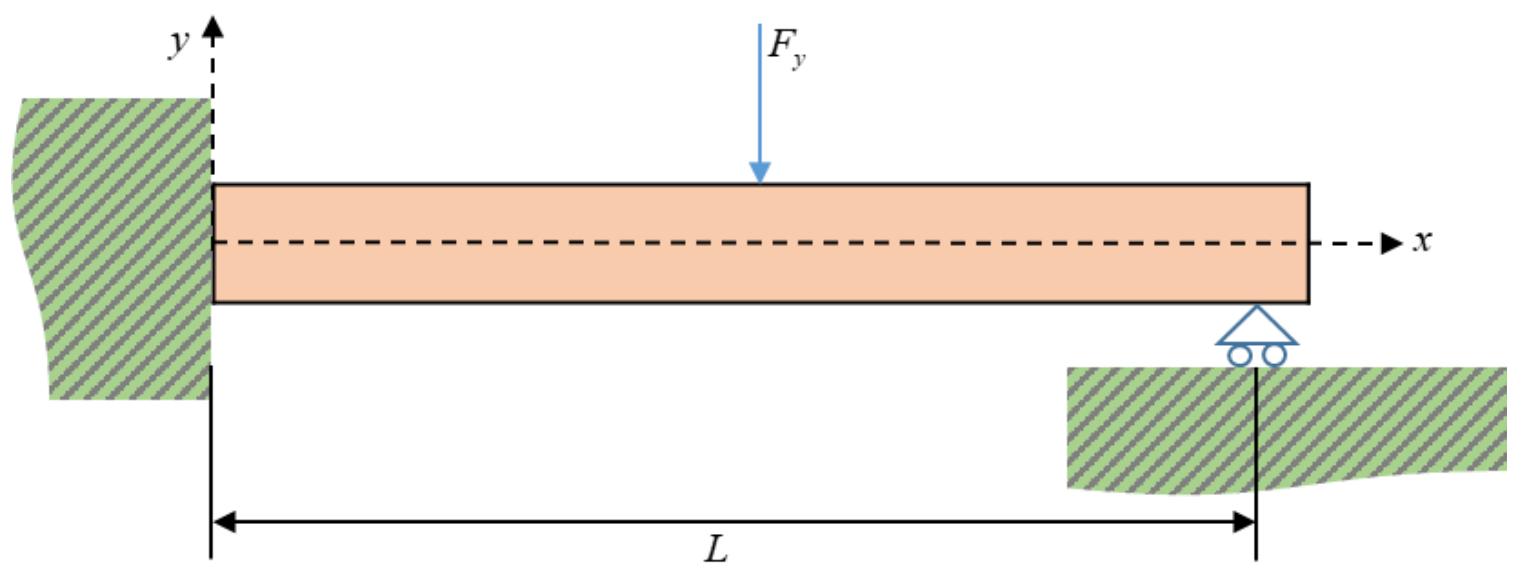


Figure 5. Clamped-simply supported beam subjected to transverse loading.

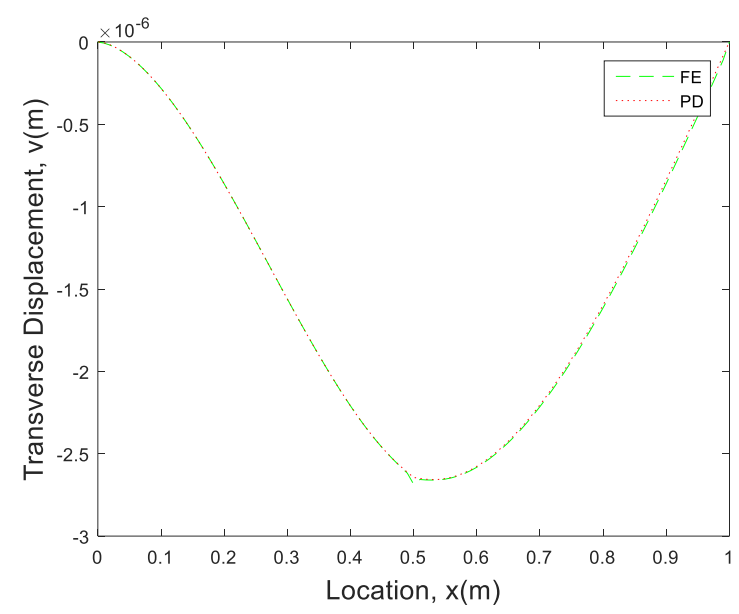

(a)

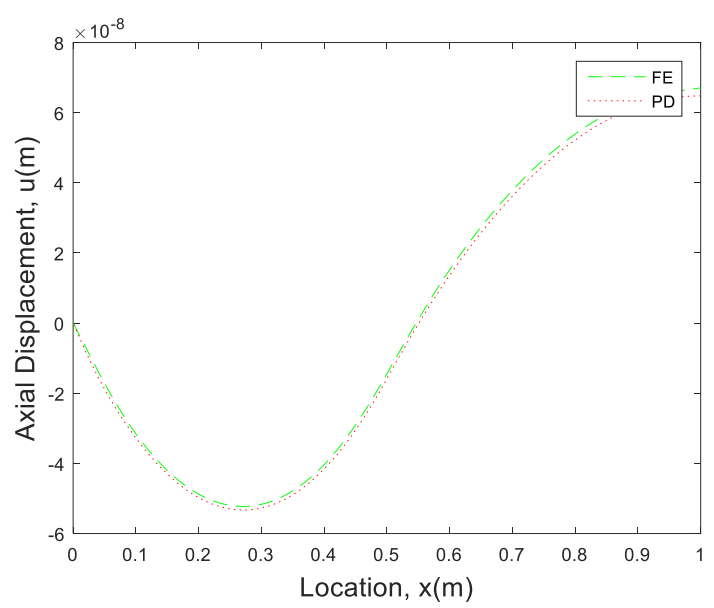

(b)

Figure 6. Variation of (a) transverse displacement, (b) axial displacement along the beam.

As in the previous cases, PD and FE results also agree with each other for this mixed (clamped-roller supported) boundary condition case for both axial and transverse displacement as shown in Fig. 6. The maximum difference between PD and FE results is 3.5\% which can be further reduced by increasing the numerical of material points in the PD solution domain.

\section{Conclusions}

In this study, a new peridynamic higher-order beam formulation for functionally graded materials was developed by using Euler-Lagrange equation. Three different numerical examples were considered for a beam subjected to simply supported, clamped and mixed boundary conditions, respectively. In all these cases, variation of axial and transverse deflections were obtained from both peridynamics and finite element analyses. A very good agreement was observed between peridynamics and finite element analysis demonstrating the accuracy of the newly developed formulation. The developed formulation can be utilised for failure analysis of functionally graded beams after incorporating a suitable failure criterion. Moreover, 
since peridynamics is a non-local continuum mechanics formulation and has a length scale parameter, i.e. horizon, the current formulation can be beneficial to represent non-classical deformation behaviour especially seen at small scales. Therefore, the developed formulation can be used for the analysis of functionally graded nanobeams.

\section{References}

M. Asghari, M. Rahaeifard, M.H. Kahrobaiyan, M.T. Ahmadian, The modified couple stress functionally graded Timoshenko beam formulation, Materials \& Design 32(3) (2011) 1435-1443.

A. Boussoula, B. Boucham, M. Bourada, F. Bourada, A. Tounsi, A.A. Bousahla, and A. Tounsi, A simple nth-order shear deformation theory for thermomechanical bending analysis of different configurations of FG sandwich plates, Smart Structures and Systems 25(2) (2020) 197-218.

F.F. Calim, Transient analysis of axially functionally graded Timoshenko beams with variable cross-section,

Composites Part B: Engineering 98 (2016) 472-483.

S.C. Chikr, A. Kaci, A.A. Bousahla, F. Bourada, A. Tounsi, E.A. Bedia, S.R. Mahmoud, K.H. Benrahou, A. Tounsi, A novel four-unknown integral model for buckling response of FG sandwich plates resting on elastic foundations under various boundary conditions using Galerkin's approach, Geomechanics and Engineering 21(5) (2020) 471-487.

C. Diyaroglu, E. Oterkus, S. Oterkus, E. Madenci, Peridynamics for bending of beams and plates with transverse shear deformation, International Journal of Solids and Structures 69 (2015) 152-168.

C. Diyaroglu, E. Oterkus, S. Oterkus, An Euler-Bernoulli beam formulation in an ordinary state-based peridynamic framework, Mathematics and Mechanics of Solids 24(2) (2019) 361-376.

F. Ebrahimi, M. Zia, Large amplitude nonlinear vibration analysis of functionally graded Timoshenko beams with porosities, Acta Astronautica 116 (2015) 117-125.

F. Ebrahimi, M.R. Barati, A nonlocal higher-order shear deformation beam theory for vibration analysis of size-dependent functionally graded nanobeams, Arabian Journal for Science and Engineering, 41(5) (2016) 1679-1690. 
J. Fariborz, R.C. Batra, Free vibration of bi-directional functionally graded material circular beams using shear deformation theory employing logarithmic function of radius, Composite Structures 210 (2019) 217-230.

M. Kaddari, A. Kaci, A.A. Bousahla, A. Tounsi, F. Bourada, A. Tounsi, E.A. Bedia, M.A. Al-Osta, A study on the structural behaviour of functionally graded porous plates on elastic foundation using a new quasi-3D model: bending and free vibration analysis, Computers and Concrete, 25(1) (2020) 37-57.

R. Kadoli, K. Akhtar, N. Ganesan, Static analysis of functionally graded beams using higher order shear deformation theory, Applied Mathematical Modelling 32(12) (2008) 2509-2525.

X.F. Li, A unified approach for analyzing static and dynamic behaviors of functionally graded Timoshenko and Euler-Bernoulli beams, Journal of Sound and Vibration 318(4-5) (2008) 1210-1229.

S.R. Li, R.C. Batra, Relations between buckling loads of functionally graded Timoshenko and homogeneous Euler-Bernoulli beams, Composite Structures, 95 (2013) 5-9.

S. Liu, G. Fang, J. Liang, M. Fu, B. Wang, A new type of peridynamics: Element-based peridynamics, Computer Methods in Applied Mechanics and Engineering 366 (2020) 113098.

E. Madenci, E. Oterkus, Peridynamic Theory and Its Applications, Springer, New York, NY, 2014.

H. Matouk, A.A. Bousahla, H. Heireche, F. Bourada, E.A. Bedia, A. Tounsi, S.R. Mahmoud, A. Tounsi, K.H. Benrahou, Investigation on hygro-thermal vibration of P-FG and symmetric S-FG nanobeam using integral Timoshenko beam theory, Advances in Nano Research, 8(4) (2020) 293-305.

A. Menasria, A. Kaci, A.A. Bousahla, F. Bourada, A. Tounsi, K.H. Benrahou, A. Tounsi, E.A. Adda Bedia, S.R. Mahmoud, A four-unknown refined plate theory for dynamic analysis of FG-sandwich plates under various boundary conditions, Steel and Composite Structures 36(3) (2020) 355-367.

J. O’Grady, J. Foster, Peridynamic beams: a non-ordinary, state-based model, International Journal of Solids and Structures, 51(18) (2014) 3177-3183.

E. Oterkus, I. Guven, E. Madenci, Impact damage assessment by using peridynamic theory, Open Engineering 2(4) (2012) 523-531.

A. Pydah, and R.C. Batra, Shear deformation theory using logarithmic function for thick circular beams and analytical solution for bi-directional functionally graded circular beams, Composite Structures 172 (2017) 45-60. 
M. Rabhi, K.H. Benrahou, A. Kaci, M.S.A. Houari, F. Bourada, A.A. Bousahla, A. Tounsi, E.A. Bedia, S.R. Mahmoud, A. Tounsi, A new innovative 3-unknowns HSDT for buckling and free vibration of exponentially graded sandwich plates resting on elastic foundations under various boundary conditions, Geomechanics and Engineering 22(2) (2020) 119.

S. Refrafi, A.A. Bousahla, A. Bouhadra, A. Menasria, F. Bourada, A. Tounsi, E.A. Bedia, S.R. Mahmoud, K.H. Benrahou, A. Tounsi, Effects of hygro-thermo-mechanical conditions on the buckling of FG sandwich plates resting on elastic foundations, Computers and Concrete 25(4) (2020) 311-325.

A. Shariati, A. Ghabussi, M. Habibi, H. Safarpour, M. Safarpour, A. Tounsi, M. Safa, Extremely large oscillation and nonlinear frequency of a multi-scale hybrid disk resting on nonlinear elastic foundation, Thin-Walled Structures 154 (2020) 106840.

S.A. Silling, Reformulation of elasticity theory for discontinuities and long-range forces, Journal of the Mechanics and Physics of Solids 48(1) (2000) 175-209.

M. Simsek, Non-linear vibration analysis of a functionally graded Timoshenko beam under action of a moving harmonic load, Composite Structures 92(10) (2010) 2532-2546.

M. Simsek, J.N. Reddy, Bending and vibration of functionally graded microbeams using a new higher order beam theory and the modified couple stress theory, International Journal of Engineering Science 64 (2013) $37-53$.

Y. Sun, S.R. Li, R.C. Batra, Thermal buckling and post-buckling of FGM Timoshenko beams on nonlinear elastic foundation, Journal of Thermal Stresses 39(1) (2016) 11-26.

S.A. Tajalli, M. Rahaeifard, M.H. Kahrobaiyan, M.R. Movahhedy, J. Akbari, M.T. Ahmadian, Mechanical behavior analysis of size-dependent micro-scaled functionally graded Timoshenko beams by strain gradient elasticity theory, Composite Structures 102 (2013) 72-80.

A. Tounsi, S.U. Al-Dulaijan, M.A. Al-Osta, A. Chikh, M.M. Al-Zahrani, A. Sharif, A. Tounsi, A four variable trigonometric integral plate theory for hygro-thermo-mechanical bending analysis of AFG ceramic-metal plates resting on a two-parameter elastic foundation, Steel and Composite Structures, 34(4) (2020) 511. Y. Tong, W. Shen, J. Shao, J. Chen, A new bond model in peridynamics theory for progressive failure in cohesive brittle materials, Engineering Fracture Mechanics 223 (2020) 106767. 
H. Wang, E. Oterkus, S. Oterkus, 2018. Predicting fracture evolution during lithiation process using peridynamics, Engineering Fracture Mechanics 192 (2018) 176-191.

Z. Yang, E. Oterkus, C.T. Nguyen, S. Oterkus, 2019. Implementation of peridynamic beam and plate formulations in finite element framework, Continuum Mechanics and Thermodynamics 31(1) (2019) $301-315$.

H. Yu, S. Li, 2020. On Energy Release Rates in Peridynamics, Journal of the Mechanics and Physics of Solids, (2020) 104024.

A. Zine, A.A. Bousahla, F. Bourada, K.H. Benrahou, A. Tounsi, E.A. Adda Bedia, S.R. Mahmoud, A. Tounsi, Bending analysis of functionally graded porous plates via a refined shear deformation theory, Computers and Concrete 26(1) (2020) 63-74.

\section{Appendix}

As derived above, the classical strain energy function of the higher order functionally graded beam can be written as

$$
\begin{aligned}
& \mathrm{W}=\frac{1}{2 A}\left\{\int_{A} \frac{E}{1-\vartheta^{2}} d A\left[\left(\frac{\partial u}{\partial x}\right)^{2}+\left(\varphi_{y}\right)^{2}\right]+\int_{A} \frac{2 E y}{1-\vartheta^{2}} d A\left(\frac{\partial u}{\partial x} \frac{\partial \varphi}{\partial x}+2 \varphi_{y} v^{*}\right)+\int_{A} \frac{E y^{2}}{1-\vartheta^{2}} d A\left[\left(\frac{\partial \varphi}{\partial x}\right)^{2}+2 \frac{\partial u}{\partial x} \frac{\partial u^{*}}{\partial x}+\right.\right. \\
& \left.4\left(v^{*}\right)^{2}\right]+\int_{A} \frac{2 E y^{3}}{1-\vartheta^{2}} d A\left(\frac{\partial u}{\partial x} \frac{\partial \varphi^{*}}{\partial x}+\frac{\partial \varphi}{\partial x} \frac{\partial u^{*}}{\partial x}\right)+\int_{A} \frac{E y^{4}}{1-\vartheta^{2}} d A\left[\left(\frac{\partial u^{*}}{\partial x}\right)^{2}+2 \frac{\partial \varphi}{\partial x} \frac{\partial \varphi^{*}}{\partial x}\right]+\int_{A} \frac{2 E y^{5}}{1-\vartheta^{2}} d A \frac{\partial u^{*}}{\partial x} \frac{\partial \varphi^{*}}{\partial x}+ \\
& \left.\int_{A} \frac{E y^{6}}{1-v^{2}} d A\left(\frac{\partial \theta^{*}}{\partial x}\right)^{2}\right\}+\left[\int_{A} \frac{E v}{1-\vartheta^{2}} d A \frac{\partial u}{\partial x} \varphi_{y}+\int_{A} \frac{E \vartheta y}{1-\vartheta^{2}} d A\left(2 \frac{\partial u}{\partial x} v^{*}+\frac{\partial \varphi}{\partial x} \varphi_{y}\right)+\int_{A} \frac{E \vartheta y^{2}}{1-\vartheta^{2}} d A\left(2 \frac{\partial \varphi}{\partial x} v^{*}+\right.\right. \\
& \left.\left.\frac{\partial u^{*}}{\partial x} \varphi_{y}\right)+\int_{A} \frac{E \vartheta y^{3}}{1-\vartheta^{2}} d A\left(2 \frac{\partial u^{*}}{\partial x} v^{*}+\frac{\partial \varphi^{*}}{\partial x} \varphi_{y}\right)+\int_{A} \frac{2 E \vartheta y^{4}}{1-\vartheta^{2}} d A \frac{\partial \varphi^{*}}{\partial x} v^{*}\right]+\frac{1}{2 A}\left\{\int_{A} \mu d A\left(\varphi+\frac{\partial v}{\partial x}\right)^{2}+\right. \\
& 2 \int_{A} \mu y d A\left(\varphi+\frac{\partial v}{\partial x}\right)\left(2 u^{*}+\frac{\partial \varphi_{y}}{\partial x}\right)+\int_{A} \mu y^{2} d A\left[\left(2 u^{*}+\frac{\partial \varphi_{y}}{\partial x}\right)^{2}+2\left(\varphi+\frac{\partial v}{\partial x}\right)\left(3 \varphi^{*}+\frac{\partial v^{*}}{\partial x}\right)\right]+ \\
& \left.2 \int_{A} \mu y^{3} d A\left(2 u^{*}+\frac{\partial \varphi_{y}}{\partial x}\right)\left(3 \theta^{*}+\frac{\partial v^{*}}{\partial x}\right)+\int_{A} \mu y^{4} d A\left(3 \varphi^{*}+\frac{\partial v^{*}}{\partial x}\right)^{2}\right\}
\end{aligned}
$$

Eq. (A1) can be separated into three parts for simplification as

$$
W=W_{I}+W_{I I}+W_{I I I}
$$


where

$W_{I}=\frac{1}{2 A}\left\{\int_{A} \frac{E}{1-\vartheta^{2}} d A\left[\left(\frac{\partial u}{\partial x}\right)^{2}+\left(\varphi_{y}\right)^{2}\right]+\int_{A} \frac{2 E y}{1-\vartheta^{2}} d A\left(\frac{\partial u}{\partial x} \frac{\partial \varphi}{\partial x}+2 \varphi_{y} v^{*}\right)+\int_{A} \frac{E y^{2}}{1-\vartheta^{2}} d A\left[\left(\frac{\partial \varphi}{\partial x}\right)^{2}+2 \frac{\partial u}{\partial x} \frac{\partial u^{*}}{\partial x}+\right.\right.$ $\left.4\left(v^{*}\right)^{2}\right]+\int_{A} \frac{2 E y^{3}}{1-\vartheta^{2}} d A\left(\frac{\partial u}{\partial x} \frac{\partial \varphi^{*}}{\partial x}+\frac{\partial \varphi}{\partial x} \frac{\partial u^{*}}{\partial x}\right)+\int_{A} \frac{E y^{4}}{1-\vartheta^{2}} d A\left[\left(\frac{\partial u^{*}}{\partial x}\right)^{2}+2 \frac{\partial \varphi}{\partial x} \frac{\partial \varphi^{*}}{\partial x}\right]+\int_{A} \frac{2 E y^{5}}{1-\vartheta^{2}} d A \frac{\partial u^{*}}{\partial x} \frac{\partial \varphi^{*}}{\partial x}+$

$\left.\int_{A} \frac{E y^{6}}{1-\vartheta^{2}} d A\left(\frac{\partial \varphi^{*}}{\partial x}\right)^{2}\right\}$

$W_{I I}=\int_{A} \frac{E \vartheta}{1-\vartheta^{2}} d A \frac{\partial u}{\partial x} \varphi_{y}+\int_{A} \frac{E \vartheta z}{1-\vartheta^{2}} d A\left(2 \frac{\partial u}{\partial x} v^{*}+\frac{\partial \varphi}{\partial x} \varphi_{y}\right)+\int_{A} \frac{E \vartheta y^{2}}{1-\vartheta^{2}} d A\left(2 \frac{\partial \varphi}{\partial x} v^{*}+\frac{\partial u^{*}}{\partial x} \varphi_{y}\right)+$

$\int_{A} \frac{E \vartheta y^{3}}{1-\vartheta^{2}} d A\left(2 \frac{\partial u^{*}}{\partial x} v^{*}+\frac{\partial \varphi^{*}}{\partial x} \varphi_{y}\right)+\int_{A} \frac{2 E \vartheta y^{4}}{1-\vartheta^{2}} d A \frac{\partial \varphi^{*}}{\partial x} v^{*}$

and

$W_{I I I}=\frac{1}{2 A}\left\{\int_{A} \mu d A\left(\varphi+\frac{\partial v}{\partial x}\right)^{2}+2 \int_{A} \mu y d A\left(\varphi+\frac{\partial v}{\partial x}\right)\left(2 u^{*}+\frac{\partial \varphi_{y}}{\partial x}\right)+\int_{A} \mu y^{2} d A\left[\left(2 u^{*}+\frac{\partial \varphi_{y}}{\partial x}\right)^{2}+\right.\right.$

$\left.\left.2\left(\varphi+\frac{\partial v}{\partial x}\right)\left(3 \varphi^{*}+\frac{\partial v^{*}}{\partial x}\right)\right]+2 \int_{A} \mu y^{3} d A\left(2 u^{*}+\frac{\partial \varphi_{y}}{\partial x}\right)\left(3 \varphi^{*}+\frac{\partial v^{*}}{\partial x}\right)+\int_{A} \mu y^{4} d A\left(3 \varphi^{*}+\frac{\partial v^{*}}{\partial x}\right)^{2}\right\}$

Next, Taylor's expansion will be utilised to determine the corresponding PD forms of Eqs. (A3a-c).

A1.1 Transforming $W_{I}$ into PD form

Following relationships can be established by using Taylor's expansion as

$u(x+\xi)-u(x)=\frac{\partial u(x)}{\partial x} \xi+0\left(\xi^{2}\right)$

$\varphi_{y}(x+\xi)=\varphi_{y}(x)+0(\xi)$

If we multiply both sides of Eq. (A4b) with $\xi$ and perform some algebraic manipulations, the following relationship can be obtained as

$\frac{\varphi_{y}(x+\xi)+\varphi_{y}(x)}{2} \xi=\varphi_{y}(x) \xi+O\left(\xi^{2}\right)$

Squaring Eqs. (A4a) and (A5) and ignoring higher order terms yield 
$[u(x+\xi)-u(x)]^{2}=\left(\frac{\partial u(x)}{\partial x}\right)^{2} \xi^{2}$

$\left(\frac{\varphi_{y}(x+\xi)+\varphi_{y}(x)}{2}\right)^{2} \xi^{2}=\left[\varphi_{y}(x)\right]^{2} \xi^{2}$

Combining Eqs. (A6a) with (A6b) and dividing each term with $|\xi|$ yields

$\frac{[u(x+\xi)-u(x)]^{2}}{|\xi|}+\left(\frac{\varphi_{y}(x+\xi)+\varphi_{y}(x)}{2}\right)^{2}|\xi|=\left\{\left(\frac{\partial u(x)}{\partial x}\right)^{2}+\left[\varphi_{y}(x)\right]^{2}\right\}|\xi|$

Integrating each terms of Eq. (A7) over the horizon gives

$\int_{-\delta}^{\delta}\left(\frac{[u(x+\xi)-u(x)]^{2}}{|\xi|}+\left(\frac{\varphi_{y}(x+\xi)+\varphi_{y}(x)}{2}\right)^{2}|\xi|\right) d \xi=\left\{\left(\frac{\partial u(x)}{\partial x}\right)^{2}+\left[\varphi_{y}(x)\right]^{2}\right\} \int_{-\delta}^{\delta}|\xi| d \xi$

which then yields

$\left(\frac{\partial u(x)}{\partial x}\right)^{2}+\left[\varphi_{y}(x)\right]^{2}=\frac{1}{\delta^{2}} \int_{-\delta}^{\delta}\left(\frac{[u(x+\xi)-u(x)]^{2}}{|\xi|}+\left(\frac{\varphi_{y}(x+\xi)+\varphi_{y}(x)}{2}\right)^{2}|\xi|\right) d \xi$

Eq. (A8b) can be written in discretized form for the material point $k$ as

$\left(\frac{\partial u_{(k)}}{\partial x}\right)^{2}+\left(\varphi_{y}^{(k)}\right)^{2}=\frac{1}{\delta^{2} A} \sum_{i}\left[\frac{\left(u_{\left.\left(i^{k}\right)^{-u_{(k)}}\right)^{2}}\right.}{\left|\xi_{\left(i^{k}\right)(k)}\right|}+\left(\frac{\varphi_{y}^{\left(i^{k}\right)}+\varphi_{y}^{(k)}}{2}\right)^{2}\left|\xi_{\left(i^{k}\right)(k)}\right|\right] V_{\left(i^{k}\right)}$

Similarly, following relationships can be established by using Taylor's expansion as

$u(x+\xi)-u(x)=\frac{\partial u(x)}{\partial x} \xi$

$\varphi(x+\xi)-\varphi(x)=\frac{\partial \varphi(x)}{\partial x} \xi$

$\frac{\varphi_{y}(x+\xi)+\varphi_{y}(x)}{2} \xi=\varphi_{y}(x) \xi$

$\left[v^{*}(x+\xi)+v^{*}(x)\right] \xi=2 v^{*}(x) \xi$

Multiplying Eq. (A10a) with Eq. (A10b) and Eq. (A10c) with Eq. (A10d) gives 
$[u(x+\xi)-u(x)][\varphi(x+\xi)-\varphi(x)]=\frac{\partial u(x)}{\partial x} \frac{\partial \varphi(x)}{\partial x} \xi^{2}$

$\left[v^{*}(x+\xi)+v^{*}(x)\right] \frac{\varphi_{y}(x+\xi)+\varphi_{y}(x)}{2} \xi^{2}=2 v^{*}(x) \varphi_{y}(x) \xi^{2}$

Combining Eq. (A11a) with Eq. (A11b) results in

$\frac{[u(x+\xi)-u(x)][\varphi(x+\xi)-\varphi(x)]}{|\xi|}+\left[v^{*}(x+\xi)+v^{*}(x)\right] \frac{\varphi_{y}(x+\xi)+\varphi_{y}(x)}{2}|\xi|=\left[\frac{\partial u(x)}{\partial x} \frac{\partial \varphi(x)}{\partial x}+2 v^{*}(x) \varphi_{y}(x)\right]|\xi|$

Integrating each term of Eq. (A12) over the horizon yields

$\frac{\partial u(x)}{\partial x} \frac{\partial \varphi(x)}{\partial x}+2 v^{*}(x) \varphi_{y}(x)=\frac{1}{\delta^{2}} \int_{-\delta}^{\delta}\left\{\frac{[u(x+\xi)-u(x)][\varphi(x+\xi)-\varphi(x)]}{|\xi|}+\left[v^{*}(x+\xi)+v^{*}(x)\right] \frac{\varphi_{y}(x+\xi)+\varphi_{y}(x)}{2}|\xi|\right\} d \xi$

Eq. (A13) can be written in discretized form for the material point $k$ as

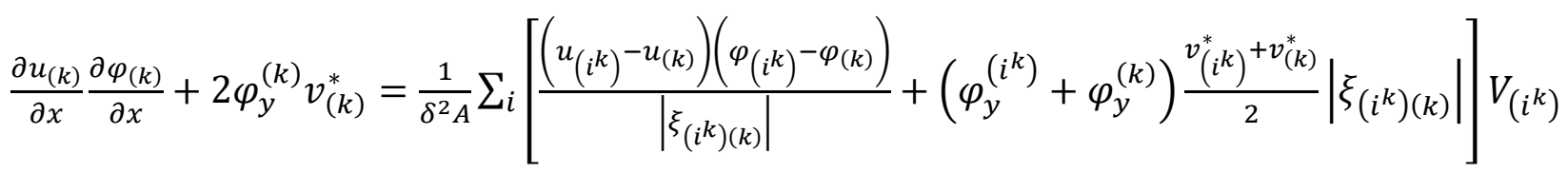

Following a similar approach, the remaining local terms of $W_{I}$ can be transformed as

$$
\begin{aligned}
& \left(\frac{\partial \varphi_{(k)}}{\partial x}\right)^{2}+2 \frac{\partial u_{(k)}}{\partial x} \frac{\partial u_{(k)}^{*}}{\partial x}+4\left(v_{(k)}^{*}\right)^{2}=\frac{1}{\delta^{2} A} \sum_{i}\left[\frac{\left(\varphi_{\left.\left(i^{k}\right)^{-\varphi_{(k)}}\right)^{2}}\right.}{\left|\xi_{\left(i^{k}\right)(k)}\right|}+2 \frac{\left(u_{\left(i^{k}\right)}-u_{(k)}\right)\left(u_{\left(i^{k}\right)}^{*}-u_{(k)}^{*}\right)}{\left|\xi_{\left(i^{k}\right)(k)}\right|}+\left(v_{\left(i^{k}\right)}^{*}+\right.\right. \\
& \left.\left.v_{(k)}^{*}\right)^{2}\left|\xi_{\left(i^{k}\right)(k)}\right|\right] V_{\left(i^{k}\right)}
\end{aligned}
$$

$\frac{\partial u_{(k)}}{\partial x} \frac{\partial \varphi_{(k)}^{*}}{\partial x}+\frac{\partial \varphi_{(k)}}{\partial x} \frac{\partial u_{(k)}^{*}}{\partial x}=\frac{1}{\delta^{2} A} \sum_{i}\left[\frac{\left.\left(u_{\left(i^{k}\right)}\right)^{\left.-u_{(k)}\right)}\right)\left(\varphi_{\left(i^{k}\right)}^{*}-\varphi_{(k)}^{*}\right)}{\left|\xi_{\left(i^{k}\right)(k)}\right|}+\frac{\left(\varphi_{\left(i^{k}\right)}-\varphi_{(k)}\right)\left(u_{\left(i^{k}\right)}^{*}-u_{(k)}^{*}\right)}{\left|\xi_{\left(i^{k}\right)(k)}\right|}\right] V_{\left(i^{k}\right)}$

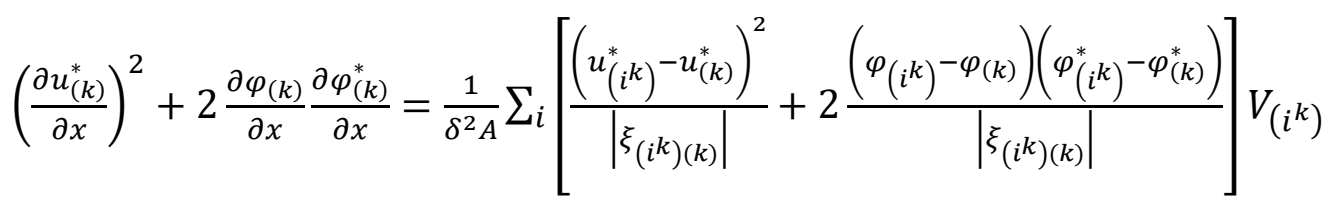




$$
\begin{aligned}
& \frac{\partial u_{(k)}^{*}}{\partial x} \frac{\partial \varphi_{(k)}^{*}}{\partial x}=\frac{1}{\delta^{2} A} \sum_{i} \frac{\left(u_{\left(i^{k}\right)}^{*}-u_{(k)}^{*}\right)\left(\varphi_{\left(i^{k}\right)}^{*}-\varphi_{(k)}^{*}\right)}{\left|\xi_{\left(i^{k}\right)(k)}\right|} V_{\left(i^{k}\right)} \\
& \left(\frac{\partial \varphi_{(k)}^{*}}{\partial x}\right)^{2}=\frac{1}{\delta^{2} A} \sum_{i} \frac{\left(\varphi_{\left(i^{k}\right)}^{*}-\varphi_{(k)}^{*}\right)^{2}}{\left|\xi_{\left(i^{k}\right)(k)}\right|} V_{\left(i^{k}\right)}
\end{aligned}
$$

Substituting Eqs. (A9), (A14) and (A15) into Eq. (A3a) results in the first part of the strain energy density function, $W_{I}$, in PD form as

$$
\begin{aligned}
& W_{I}^{(k)}=\frac{1}{2} \frac{1}{\delta^{2} A^{2}}\left\{\int_{A} \frac{E}{1-\vartheta^{2}} d A \sum_{i}\left[\frac{\left(u_{\left(i^{k}\right)^{-}}\left(u_{(k)}\right)^{2}\right.}{\left|\xi_{\left(i^{k}\right)(k)}\right|}+\left(\frac{\varphi_{y}^{\left(i^{k}\right)}+\varphi_{y}^{(k)}}{2}\right)^{2}\left|\xi_{\left(i^{k}\right)(k)}\right|\right] V_{\left(i^{k}\right)}+\right. \\
& \int_{A} \frac{2 E y}{1-\vartheta^{2}} d A \sum_{i}\left[\frac{\left(u_{\left(i^{k}\right)}-u_{(k)}\right)\left(\varphi_{\left(i^{k}\right)}-\varphi_{(k)}\right)}{\left|\xi_{\left(i^{k}\right)(k)}\right|}+\left(\varphi_{y}^{\left(i^{k}\right)}+\varphi_{y}^{(k)}\right) \frac{v_{\left(i^{k}\right)}^{*}+v_{(k)}^{*}}{2}\left|\xi_{\left(i^{k}\right)(k)}\right|\right] V_{\left(i^{k}\right)}+ \\
& \int_{A} \frac{E y^{2}}{1-\vartheta^{2}} d A \sum_{i}\left[\frac{\left(\varphi_{\left(i^{k}\right)^{-}}\left(\varphi_{(k)}\right)^{2}\right.}{\left|\xi_{\left(i^{k}\right)(k)}\right|}+2 \frac{\left.\left(u_{\left(i^{k}\right)}\right)^{\left.-u_{(k)}\right)}\right)\left(u_{\left(i^{k}\right)}^{*}\right)^{\left.-u_{(k)}^{*}\right)}}{\left|\xi_{\left(i^{k}\right)(k)}\right|}+\left(v_{\left(i^{k}\right)}^{*}+v_{(k)}^{*}\right)^{2}\left|\xi_{\left(i^{k}\right)(k)}\right|\right] V_{\left(i^{k}\right)}+ \\
& \int_{A} \frac{2 E y^{3}}{1-\vartheta^{2}} d A \sum_{i}\left[\frac{\left.\left.\left(u_{\left(i^{k}\right)}\right)_{(k)}\right)\left(\varphi_{\left(i^{k}\right)}^{*}\right)_{(k)}^{*}\right)}{\left|\xi_{\left(i^{k}\right)(k)}\right|}+\frac{\left(\varphi_{\left(i^{k}\right)}-\varphi_{(k)}\right)\left(u_{\left(i^{k}\right)}^{*}-u_{(k)}^{*}\right)}{\left|\xi_{\left(i^{k}\right)(k)}\right|}\right] V_{\left(i^{k}\right)}+\int_{A} \frac{E y^{4}}{1-\vartheta^{2}} d A \sum_{i}\left[\frac{\left(u_{\left(i^{k}\right)^{*}}-u_{(k)}^{*}\right)^{2}}{\left|\xi_{\left(i^{k}\right)(k)}\right|}+\right. \\
& \left.2 \frac{\left.\left(\varphi_{\left(i^{k}\right)}\right)_{(k)}\right)\left(\varphi_{\left(i^{k}\right)}^{*}-\varphi_{(k)}^{*}\right)}{\left|\xi_{\left(i^{k}\right)(k)}\right|}\right] V_{\left(i^{k}\right)}+\int_{A} \frac{2 E y^{5}}{1-\vartheta^{2}} d A \sum_{i} \frac{\left(u_{\left(i^{k}\right)}^{*}-u_{(k)}^{*}\right)\left(\varphi_{\left(i^{k}\right)}^{*}-\varphi_{(k)}^{*}\right)}{\left|\xi_{\left(i^{k}\right)(k)}\right|} V_{\left(i^{k}\right)}+
\end{aligned}
$$$$
\left.\int_{A} \frac{E y^{6}}{1-\vartheta^{2}} d A \sum_{i} \frac{\left(\varphi_{\left(i^{k}\right)}^{*}-\varphi_{(k)}^{*}\right)^{2}}{\left|\xi_{\left(i^{k}\right)(k)}\right|} V_{\left(i^{k}\right)}\right\}
$$

\section{A1.2 Transform $W_{I I}$ into PD form}

Following relationships can be established by using Taylor's expansion as

$$
\begin{aligned}
& u(x+\xi)-u(x)=\frac{\partial u(x)}{\partial x} \xi \\
& \frac{\varphi_{y}(x+\xi)+\varphi_{y}(x)}{2} \xi=\varphi_{y}(x) \xi
\end{aligned}
$$


Multiplying Eq. (A17a) with Eq. (A17b) yields

$[u(x+\xi)-u(x)] \frac{\varphi_{y}(x+\xi)+\varphi_{y}(x)}{2} \operatorname{sign}(\xi)=\frac{\partial u(x)}{\partial x} \varphi_{y}(x)|\xi|$

Integrating each terms of Eq. (A18) over the horizon yields

$\frac{\partial u(x)}{\partial x} \varphi_{y}(x)=\frac{1}{\delta^{2}} \int_{-\delta}^{\delta}[u(x+\xi)-u(x)] \frac{\varphi_{y}(x+\xi)+\varphi_{y}(x)}{2} \operatorname{sign}(\xi) d \xi$

Eq. (A19) can be written in discretized form for the material point $k$ as

$\frac{\partial u_{(k)}}{\partial x} \varphi_{y}^{(k)}=\frac{1}{\delta^{2} A} \sum_{i}\left(u_{\left(i^{k}\right)}-u_{(k)}\right) \frac{\varphi_{y}^{\left(i^{k}\right)}+\varphi_{y}^{(k)}}{2} \operatorname{sign}\left(\xi_{\left(i^{k}\right)(k)}\right) V_{\left(i^{k}\right)}$

The remaining local terms of $W_{I I}$ can be written by following a similar approach as

$2 v_{(k)}^{*} \frac{\partial u_{(k)}}{\partial x}+\varphi_{y}^{(k)} \frac{\partial \varphi_{(k)}}{\partial x}=\frac{1}{\delta^{2} A} \sum_{i}\left[\left(u_{\left(i^{k}\right)}-u_{(k)}\right)\left(v_{\left(i^{k}\right)}^{*}+v_{(k)}^{*}\right) \operatorname{sign}\left(\xi_{\left(i^{k}\right)(k)}\right)+\left(\varphi_{\left(i^{k}\right)}-\right.\right.$

$\left.\left.\varphi_{(k)}\right) \frac{\varphi_{y}^{\left(i^{k}\right)}+\varphi_{y}^{(k)}}{2} \operatorname{sign}\left(\xi_{\left(i^{k}\right)(k)}\right)\right] V_{\left(i^{k}\right)}$

$2 v_{(k)}^{*} \frac{\partial \varphi_{(k)}}{\partial x}+\varphi_{y}^{(k)} \frac{\partial u_{(k)}^{*}}{\partial x}=\frac{1}{\delta^{2} A} \sum_{i}\left[\left(\varphi_{\left(i^{k}\right)}-\varphi_{(k)}\right)\left(w_{\left(i^{k}\right)}^{*}+w_{(k)}^{*}\right) \operatorname{sign}\left(\xi_{\left(i^{k}\right)(k)}\right)+\left(u_{\left(i^{k}\right)}^{*}-\right.\right.$

$\left.\left.u_{(k)}^{*}\right) \frac{\varphi_{y}^{\left(i^{k}\right)}+\varphi_{y}^{(k)}}{2} \operatorname{sign}\left(\xi_{\left(i^{k}\right)(k)}\right)\right] V_{\left(i^{k}\right)}$

$2 v_{(k)}^{*} \frac{\partial u_{(k)}^{*}}{\partial x}+\varphi_{y}^{(k)} \frac{\partial \varphi_{(k)}^{*}}{\partial x}=\frac{1}{\delta^{2} A} \sum_{i}\left[\left(u_{\left(i^{k}\right)}^{*}-u_{(k)}^{*}\right)\left(v_{\left(i^{k}\right)}^{*}+v_{(k)}^{*}\right) \operatorname{sign}\left(\xi_{\left(i^{k}\right)(k)}\right)+\left(\varphi_{\left(i^{k}\right)}^{*}-\right.\right.$

$\left.\left.\varphi_{(k)}^{*}\right) \frac{\varphi_{y}^{\left(i^{k}\right)}+\varphi_{y}^{(k)}}{2} \operatorname{sign}\left(\xi_{\left(i^{k}\right)(k)}\right)\right] V_{\left(i^{k}\right)}$

$v_{(k)}^{*} \frac{\partial \varphi_{(k)}^{*}}{\partial x}=\frac{1}{\delta^{2} A} \sum_{i}\left(\varphi_{\left(i^{k}\right)}^{*}-\varphi_{(k)}^{*}\right) \frac{v_{\left(i^{k}\right)}^{*}+v_{(k)}^{*}}{2} \operatorname{sign}\left(\xi_{\left(i^{k}\right)(k)}\right) V_{\left(i^{k}\right)}$

Substituting Eqs. (A20) and (A21) into Eq. (A3b) results in the second part of strain energy density function, $W_{I I}$, in PD form as 


$$
\begin{aligned}
& W_{I I}^{(k)}=\frac{1}{\delta^{2} A^{2}}\left\{\int_{A} \frac{E \vartheta}{1-\vartheta^{2}} d A \sum_{i}\left(u_{\left(i^{k}\right)}-u_{(k)}\right) \frac{\varphi_{y}^{\left(i^{k}\right)}+\varphi_{y}^{(k)}}{2} \operatorname{sign}\left(\xi_{\left(i^{k}\right)(k)}\right) V_{\left(i^{k}\right)}+\int_{Z} \frac{E \vartheta y}{1-\vartheta^{2}} d A \sum_{i}\left[\left(u_{\left(i^{k}\right)}-\right.\right.\right. \\
& \left.\left.u_{(k)}\right)\left(v_{\left(i^{k}\right)}^{*}+v_{(k)}^{*}\right) \operatorname{sign}\left(\xi_{\left(i^{k}\right)(k)}\right)+\left(\varphi_{\left(i^{k}\right)}-\varphi_{(k)}\right) \frac{\varphi_{y}^{\left(i^{k}\right)}+\varphi_{y}^{(k)}}{2} \operatorname{sign}\left(\xi_{\left(i^{k}\right)(k)}\right)\right] V_{\left(i^{k}\right)}+ \\
& \int_{A} \frac{E \vartheta y^{2}}{1-\vartheta^{2}} d A \sum_{i}\left[\left(\theta_{\left(i^{k}\right)}-\theta_{(k)}\right)\left(v_{\left(i^{k}\right)}^{*}+v_{(k)}^{*}\right) \operatorname{sign}\left(\xi_{\left(i^{k}\right)(k)}\right)+\left(u_{\left(i^{k}\right)}^{*}-\right.\right. \\
& \left.\left.u_{(k)}^{*}\right) \frac{\varphi_{y}^{\left(i^{k}\right)}+\varphi_{y}^{(k)}}{2} \operatorname{sign}\left(\xi_{\left(i^{k}\right)(k)}\right)\right] V_{\left(i^{k}\right)}+\int_{A} \frac{E \vartheta y^{3}}{1-\vartheta^{2}} d A \sum_{i}\left[\left(u_{\left(i^{k}\right)}^{*}-u_{(k)}^{*}\right)\left(v_{\left(i^{k}\right)}^{*}+v_{(k)}^{*}\right) \operatorname{sign}\left(\xi_{\left(i^{k}\right)(k)}\right)+\right. \\
& \left.\left(\varphi_{\left(i^{k}\right)}^{*}-\varphi_{(k)}^{*}\right) \frac{\varphi_{y}^{\left(i^{k}\right)}+\varphi_{y}^{(k)}}{2} \operatorname{sign}\left(\xi_{\left(i^{k}\right)(k)}\right)\right] V_{\left(i^{k}\right)}+\int_{A} \frac{2 E \vartheta y^{4}}{1-\vartheta^{2}} d A \sum_{i}\left(\varphi_{\left(i^{k}\right)}^{*}-\right. \\
& \left.\left.\varphi_{(k)}^{*}\right) \frac{v_{\left(i^{k}\right)}^{*}+v_{(k)}^{*}}{2} \operatorname{sign}\left(\xi_{\left(i^{k}\right)(k)}\right) V_{\left(i^{k}\right)}\right\}
\end{aligned}
$$

\section{A1.3 Transforming $W_{I I I}$ into PD form}

By following a similar approach as above, local terms in $W_{I I I}$ can be transformed into PD form as

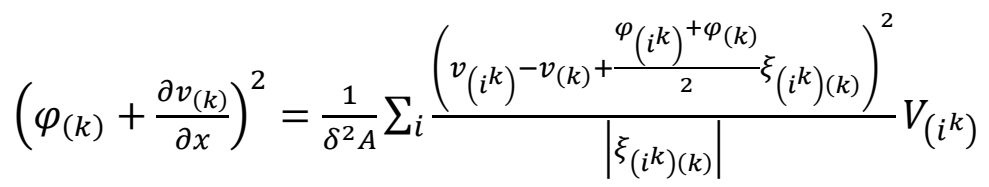

$\left(\varphi_{(k)}+\frac{\partial v_{(k)}}{\partial x}\right)\left(2 u_{(k)}^{*}+\frac{\partial \varphi_{y}^{(k)}}{\partial x}\right)=\frac{1}{\delta^{2} A} \sum_{i}\left(v_{\left(i^{k}\right)}-v_{(k)}+\frac{\varphi_{\left(i^{k}\right)}+\varphi_{(k)}}{2} \xi_{\left(i^{k}\right)(k)}\right)\left(\frac{\varphi_{y}^{\left(i^{k}\right)}-\varphi_{y}^{(k)}}{\left|\xi_{\left(i^{k}\right)(k)}\right|}+\left(u_{\left(i^{k}\right)}^{*}+\right.\right.$

$\left.\left.u_{(k)}^{*}\right) \operatorname{sign}\left(\xi_{\left(i^{k}\right)(k)}\right)\right) V_{\left(i^{k}\right)}$

$\left(\frac{\partial \varphi_{y}^{(k)}}{\partial x}+2 u_{(k)}^{*}\right)^{2}+2\left(\varphi_{(k)}+\frac{\partial v_{(k)}}{\partial x}\right)\left(3 \varphi_{(k)}^{*}+\frac{\partial v_{(k)}^{*}}{\partial x}\right)=$

$\frac{1}{\delta^{2} A} \sum_{i} \frac{\left[\varphi_{y}^{\left(i^{k}\right)}-\varphi_{y}^{(k)}+\left(u_{\left(i^{k}\right)}^{*}+u_{(k)}^{*}\right) \xi_{\left(i^{k}\right)(k)}\right]^{2}+2\left(v_{\left(i^{k}\right)}-v_{(k)}+\frac{\varphi_{\left(i^{k}\right)}+\varphi_{(k)}}{2} \xi_{\left(i^{k}\right)(k)}\right)\left(v_{\left(i^{k}\right)}^{*} v_{(k)}^{*}+3 \frac{\left.\varphi_{\left(i^{k}\right)}^{*}\right)^{+\varphi_{(k)}^{*}}}{2} \xi_{\left(i^{k}\right)(k)}\right)}{\left|\xi_{\left(i^{k}\right)(k)}\right|} V_{\left(i^{k}\right)}$ 


$$
\begin{aligned}
& \left(2 u_{(k)}^{*}+\frac{\partial \varphi_{y}^{(k)}}{\partial x}\right)\left(3 \varphi_{(k)}^{*}+\frac{\partial v_{(k)}^{*}}{\partial x}\right)=\frac{1}{\delta^{2} A} \sum_{i} \frac{\varphi_{y}^{\left(i^{k}\right)}-\varphi_{y}^{(k)}+\left(u_{\left(i^{k}\right)}^{*}+u_{(k)}^{*}\right) \xi_{\left(i^{k}\right)(k)}}{\left|\xi_{\left(i^{k}\right)(k)}\right|}\left(v_{\left(i^{k}\right)}^{*}-v_{(k)}^{*}+\right. \\
& \left.3 \frac{\varphi_{\left(i^{k}\right)}^{*}+\varphi_{(k)}^{*}}{2} \xi_{\left(i^{k}\right)(k)}\right) V_{\left(i^{k}\right)} \\
& \left(3 \varphi_{(k)}^{*}+\frac{\partial v_{(k)}^{*}}{\partial x}\right)^{2}=\frac{1}{\delta^{2} A} \sum_{i} \frac{\left.\left(v_{\left(i^{k}\right)}^{*}\right)_{(k)}^{*}+3 \frac{\left.\varphi_{\left(i^{k}\right)}^{*}\right)_{\left(\varphi_{(k)}^{*}\right.}^{*}}{2}\left(i^{k}\right)(k)\right)^{2}}{\left|\xi_{\left(i^{k}\right)(k)}\right|} V_{\left(i^{k}\right)}
\end{aligned}
$$

Substituting the equations given above into Eq. (ABc) allows $W_{I I I}$ to be written in PD form as

$$
\begin{aligned}
& W_{I I I}^{(k)}=\frac{1}{2} \frac{1}{\delta^{2} A^{2}}\left\{\int_{A} \mu d A \sum_{i} \frac{\left.\left(v_{\left(i^{k}\right)}\right)^{-v_{(k)}+\frac{\left.\varphi_{\left(i^{k}\right)}\right)^{+\varphi_{(k)}}}{2} \xi_{\left(i^{k}\right)(k)}}\right)^{2}}{\left|\xi_{\left(i^{k}\right)(k)}\right|} V_{\left(i^{k}\right)}+2 \int_{A} \mu y d A \sum_{i}\left(v_{\left(i^{k}\right)}-v_{(k)}+\right.\right. \\
& \left.\frac{\varphi_{\left(i^{k}\right)}+\varphi_{(k)}}{2} \xi_{\left(i^{k}\right)(k)}\right)\left(\frac{\varphi_{y}^{\left(i^{k}\right)}-\varphi_{y}^{(k)}}{\left|\xi_{\left(i^{k}\right)(k)}\right|}+\left(u_{\left(i^{k}\right)}^{*}+u_{(k)}^{*}\right) \operatorname{sign}\left(\xi_{\left(i^{k}\right)(k)}\right)\right) V_{\left(i^{k}\right)}+ \\
& \int_{A} \mu y^{2} d A \sum_{i} \frac{\left[\varphi_{y}^{\left(i^{k}\right)}-\varphi_{y}^{(k)}+\left(u_{\left(i^{k}\right)}^{*}+u_{(k)}^{*}\right) \xi_{\left(i^{k}\right)(k)}\right]^{2}+2\left(v_{\left(i^{k}\right)}-v_{(k)}+\frac{\varphi_{\left(i^{k}\right)}+\varphi_{(k)}}{2} \xi_{\left(i^{k}\right)(k)}\right)\left(v_{\left(i^{k}\right)}^{*}-v_{(k)}^{*}+3 \frac{\varphi_{\left(i^{k}\right)}^{*}+\varphi_{(k)}^{*}}{2} \xi_{\left(i^{k}\right)(k)}\right)}{\left|\xi_{\left(i^{k}\right)(k)}\right|} V_{\left(i^{k}\right)}+ \\
& 2 \int_{A} \mu y^{3} d A \sum_{i} \frac{\varphi_{y}^{\left(i^{k}\right)}-\varphi_{y}^{(k)}+\left(u_{\left(i^{k}\right)}^{*}+u_{(k)}^{*}\right) \xi_{\left(i^{k}\right)(k)}}{\left|\xi_{\left(i^{k}\right)(k)}\right|}\left(v_{\left(i^{k}\right)}^{*}-v_{(k)}^{*}+3 \frac{\varphi_{\left(i^{k}\right)}^{*}+\varphi_{(k)}^{*}}{2} \xi_{\left(i^{k}\right)(k)}\right) V_{\left(i^{k}\right)}+ \\
& \left.\int_{A} \mu y^{4} d A \sum_{i} \frac{\left(v_{\left(i^{k}\right)}^{*} v_{(k)}^{*}+3 \frac{\varphi_{\left(i^{k}\right)}^{*}+\varphi_{(k)}^{*}}{2} \xi_{\left(i^{k}\right)(k)}\right)^{2}}{\left|\xi_{\left(i^{k}\right)(k)}\right|} V_{\left(i^{k}\right)}\right\}
\end{aligned}
$$

Inserting Eqs. (A16), (A22) and (A24) into Eq. (A2) yields

$$
\begin{aligned}
& W_{(k)}=\frac{1}{2} \frac{1}{\delta^{2} A^{2}}\left\{\int_{A} \frac{E}{1-\vartheta^{2}} d A \sum_{i}\left[\frac{\left(u_{\left(i^{k}\right)}-u_{(k)}\right)^{2}}{\left|\xi_{\left(i^{k}\right)(k)}\right|}+\left(\frac{\varphi_{y}^{\left(i^{k}\right)}+\varphi_{y}^{(k)}}{2}\right)^{2}\left|\xi_{\left(i^{k}\right)(k)}\right|\right] V_{\left(i^{k}\right)}+\right. \\
& \int_{A} \frac{2 E z}{1-\vartheta^{2}} d A \sum_{i}\left[\frac{\left(u_{\left(i^{k}\right)}-u_{(k)}\right)\left(\varphi_{\left.\left(i^{k}\right)^{-\varphi}\right)}\right.}{\left|\xi_{\left(i^{k}\right)(k)}\right|}+\left(\varphi_{y}^{\left(i^{k}\right)}+\varphi_{y}^{(k)}\right) \frac{v_{\left(i^{k}\right)}^{*}+v_{(k)}^{*}}{2}\left|\xi_{\left(i^{k}\right)(k)}\right|\right] V_{\left(i^{k}\right)}+
\end{aligned}
$$

33 


$$
\begin{aligned}
& \int_{A} \frac{E y^{2}}{1-\vartheta^{2}} d A \sum_{i}\left[\frac{\left(\varphi_{\left.\left(i^{k}\right)^{-\varphi_{(k)}}\right)^{2}}\right.}{\left|\xi_{\left(i^{k}\right)(k)}\right|}+2 \frac{\left(u_{\left(i^{k}\right)}-u_{(k)}\right)\left(u_{\left(i^{k}\right)^{*}}^{\left.-u_{(k)}^{*}\right)}\right.}{\left|\xi_{\left(i^{k}\right)(k)}\right|}+\left(v_{\left(i^{k}\right)}^{*}+v_{(k)}^{*}\right)^{2}\left|\xi_{\left(i^{k}\right)(k)}\right|\right] V_{\left(i^{k}\right)}+ \\
& \int_{A} \frac{2 E y^{3}}{1-\vartheta^{2}} d A \sum_{i}\left[\frac{\left.\left(u_{\left(i^{k}\right)}\right)_{((k)}\right)\left(\varphi_{\left(i^{k}\right)}^{*}-\varphi_{(k)}^{*}\right)}{\left|\xi_{\left(i^{k}\right)(k)}\right|}+\frac{\left.\left(\varphi_{\left(i^{k}\right)}-\varphi_{(k)}\right)\left(u_{\left(i^{k}\right)}^{*}\right)_{(k)}^{*}\right)}{\left|\xi_{\left(i^{k}\right)(k)}\right|}\right] V_{\left(i^{k}\right)}+\int_{A} \frac{E y^{4}}{1-\vartheta^{2}} d A \sum_{i}\left[\frac{\left(u_{\left(i^{k}\right)}^{*}-u_{(k)}^{*}\right)^{2}}{\left|\xi_{\left(i^{k}\right)(k)}\right|}+\right. \\
& \left.2 \frac{\left(\varphi_{\left(i^{k}\right)}-\varphi_{(k)}\right)\left(\varphi_{\left(i^{k}\right)}^{*}-\varphi_{(k)}^{*}\right)}{\left|\xi_{(i k)(k)}\right|}\right] V_{\left(i^{k}\right)}+\int_{A} \frac{2 E y^{5}}{1-\vartheta^{2}} d A \sum_{i} \frac{\left(u_{\left(i^{k}\right)}^{*}-u_{(k)}^{*}\right)\left(\varphi_{\left(i^{k}\right)}^{*}-\varphi_{(k)}^{*}\right)}{\left|\xi_{\left(i^{k}\right)(k)}\right|} V_{\left(i^{k}\right)}+ \\
& \left.\int_{A} \frac{E y^{6}}{1-\vartheta^{2}} d A \sum_{i} \frac{\left(\varphi_{\left(i^{k}\right)^{*}}^{-\varphi_{(k)}^{*}}\right)^{2}}{\left|\xi_{\left(i^{k}\right)(k)}\right|} V_{\left(i^{k}\right)}\right\}+\frac{1}{\delta^{2} A^{2}}\left\{\int_{A} \frac{E \vartheta}{1-\vartheta^{2}} d A \sum_{i}\left(u_{\left(i^{k}\right)}-u_{(k)}\right) \frac{\varphi_{y}^{\left(i^{k}\right)}+\varphi_{y}^{(k)}}{2} \operatorname{sign}\left(\xi_{\left(i^{k}\right)(k)}\right) V_{\left(i^{k}\right)}+\right. \\
& \int_{A} \frac{E \vartheta y}{1-\vartheta^{2}} d A \sum_{i}\left[\left(u_{\left(i^{k}\right)}-u_{(k)}\right)\left(v_{\left(i^{k}\right)}^{*}+v_{(k)}^{*}\right) \operatorname{sign}\left(\xi_{\left(i^{k}\right)(k)}\right)+\left(\varphi_{\left(i^{k}\right)}-\right.\right. \\
& \left.\left.\varphi_{(k)}\right) \frac{\varphi_{y}^{\left(i^{k}\right)}+\varphi_{y}^{(k)}}{2} \operatorname{sign}\left(\xi_{\left(i^{k}\right)(k)}\right)\right] V_{\left(i^{k}\right)}+\int_{A} \frac{E \vartheta y^{2}}{1-\vartheta^{2}} d A \sum_{i}\left[\left(\varphi_{\left(i^{k}\right)}-\varphi_{(k)}\right)\left(v_{\left(i^{k}\right)}^{*}+v_{(k)}^{*}\right) \operatorname{sign}\left(\xi_{\left(i^{k}\right)(k)}\right)+\right. \\
& \left.\left(u_{\left(i^{k}\right)}^{*}-u_{(k)}^{*}\right) \frac{\varphi_{y}^{\left(i^{k}\right)}+\varphi_{y}^{(k)}}{2} \operatorname{sign}\left(\xi_{\left(i^{k}\right)(k)}\right)\right] V_{\left(i^{k}\right)}+\int_{A} \frac{E \vartheta y^{3}}{1-\vartheta^{2}} d A \sum_{i}\left[( u _ { ( i ^ { k } ) } ^ { * } - u _ { ( k ) } ^ { * } ) \left(v_{\left(i^{k}\right)}^{*}+\right.\right. \\
& \left.\left.v_{(k)}^{*}\right) \operatorname{sign}\left(\xi_{\left(i^{k}\right)(k)}\right)+\left(\varphi_{\left(i^{k}\right)}^{*}-\varphi_{(k)}^{*}\right) \frac{\varphi_{y}^{\left(i^{k}\right)}+\varphi_{y}^{(k)}}{2} \operatorname{sign}\left(\xi_{\left(i^{k}\right)(k)}\right)\right] V_{\left(i^{k}\right)}+\int_{A} \frac{2 E \vartheta y^{4}}{1-\vartheta^{2}} d A \sum_{i}\left(\varphi_{\left(i^{k}\right)}^{*}-\right.
\end{aligned}
$$

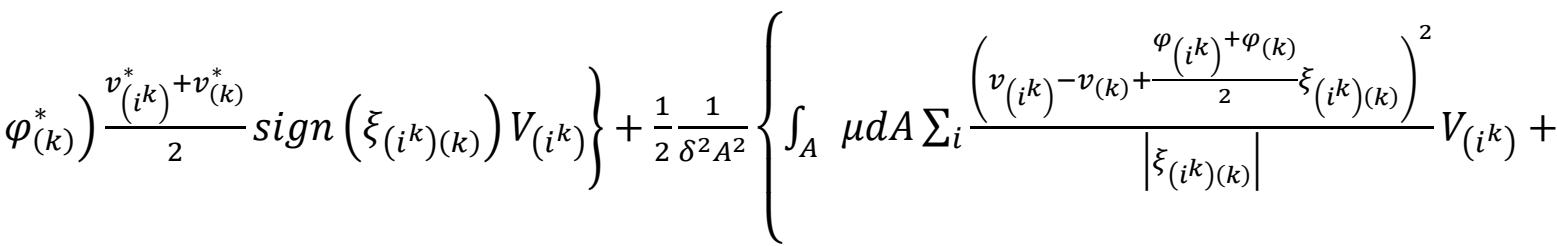

$$
\begin{aligned}
& 2 \int_{A} \operatorname{\mu ydA} \sum_{i}\left(v_{\left(i^{k}\right)}-v_{(k)}+\frac{\varphi_{\left(i^{k}\right)}+\varphi_{(k)}}{2} \xi_{\left(i^{k}\right)(k)}\right)\left(\frac{\varphi_{y}^{\left(i^{k}\right)}-\varphi_{y}^{(k)}}{\left|\xi_{\left(i^{k}\right)(k)}\right|}+\left(u_{\left(i^{k}\right)}^{*}+u_{(k)}^{*}\right) \operatorname{sign}\left(\xi_{\left(i^{k}\right)(k)}\right)\right) V_{\left(i^{k}\right)}+ \\
& \int_{A} \mu y^{2} d A \sum_{i} \frac{\left.\left[\varphi_{y}^{\left(i^{k}\right)}-\varphi_{y}^{(k)}+\left(u_{\left(i^{k}\right)}^{*}+u_{(k)}^{*}\right) \xi_{\left(i^{k}\right)(k)}\right]^{2}+2\left(v_{\left(i^{k}\right)}-v_{(k)}+\frac{\varphi_{\left(i^{k}\right)}+\varphi_{(k)}}{2} \xi_{\left(i^{k}\right)(k)}\right)\left(v_{\left(i^{k}\right)}^{*}\right)_{(k)+3}^{*} \frac{\varphi_{\left(i^{k}\right)}^{*}+\varphi_{(k)}^{*}}{2} \xi_{\left(i^{k}\right)(k)}\right)}{\left|\xi_{\left(i^{k}\right)(k)}\right|} V_{\left(i^{k}\right)}+
\end{aligned}
$$

34 
$2 \int_{A} \mu y^{3} d A \sum_{i} \frac{\varphi_{y}^{\left(i^{k}\right)}-\varphi_{y}^{(k)}+\left(u_{\left(i^{k}\right)}^{*}+u_{(k)}^{*}\right) \xi_{\left(i^{k}\right)(k)}}{\left|\xi_{\left(i^{k}\right)(k)}\right|}\left(v_{\left(i^{k}\right)}^{*}-v_{(k)}^{*}+3 \frac{\varphi_{\left(i^{k}\right)}^{*}+\varphi_{(k)}^{*}}{2} \xi_{\left(i^{k}\right)(k)}\right) V_{\left(i^{k}\right)}+$

$\left.\int_{A} \mu y^{4} d A \sum_{i} \frac{\left(v_{\left(i^{k}\right)}^{*}-v_{(k)}^{*}+3 \frac{\varphi_{\left(i^{k}\right)}^{*}+\varphi_{(k)}^{*}}{2} \xi_{\left(i^{k}\right)(k)}\right)^{2}}{\left|\xi_{\left(i^{k}\right)(k)}\right|} V_{\left(i^{k}\right)}\right\}$ 